\title{
Evolution of nanostructure and mechanical properties of silver nano-particle in the confined region between graphene sheets: An atomistic investigation
}

\author{
Sunil Kumar ${ }^{\mathrm{a}, *}$, Suchandan K. Das ${ }^{\mathrm{a}}$, Sudip K Pattanayek ${ }^{\mathrm{b}}$ \\ ${ }^{\text {a } C S I R-N a t i o n a l ~ M e t a l l u r g i c a l ~ L a b o r a t o r y, ~ J a m s h e d p u r ~ 831007, ~ I n d i a ~}$ \\ ${ }^{\mathrm{b}}$ Department of Chemical Engineering, IIT Delhi, 110016, India
}

\section{A R T I C L E I N F O}

\section{Keywords:}

Silver nano-particle

Graphene substrate

Molecular dynamics simulations

LAMMPS

Dimension-less aspect ratio

Confined region

\begin{abstract}
A B S T R A C T
Solidification and organization of silver nano-particle in a confined region between graphene sheets, shows much importance for the various application in the field of biomedical, electrochemical, coating materials, catalyst, metal-matrix nanocomposite etc. To understand the processes involved, we have studied the atomistic behaviour of solidification, organizations and mechanical properties of silver nano-particle in the bulk and as well as in confined region by molecular dynamics simulations. In the bulk, silver nano-particle shows phase transition from liquid to crystalline phase at a temperature, $\mathrm{T} \approx 1030 \pm 25 \mathrm{~K}$. However, in the confined region, silver nano-particle depicts the same phase transition at a relatively higher temperature. The tensile stress, initiation of cracks and subsequent detachment of silver during tensile deformation depends upon the temperature and interfacial interactions. The tuning of 12-6 Lennard Jones interaction potential energy parameter between graphene and silver $\left(\varepsilon_{A g-C}\right)$ drastically influenced the phase transition of silver nano-particle in the confined region. At a high interaction potential energy $\left(\varepsilon_{A g-C}\right)$, silver nano-particle shows good wettability over the graphene sheets and depicts the phase transition at a higher temperature compared to lower interaction potential energy.
\end{abstract}

\section{Introduction}

Solidification and organization of metallic materials in the confined region has been found to direct impact many desirable properties of metal matrix nano-composite, including mechanical $[1,2]$, thermal [3-5], electrical [6-8], optical [9-10], anti-microbial [11-13], etc. The accountable factors which direct the above properties are the interactions and wettability of metal atoms with interface, stress transfer, the mobility of thermal vibrations, electronic conductions and chemical reactivity. In order to understand, the appropriate reasons behind the above influenced behaviour of properties, it is much needed to study the solidification and organization of metallic material in a confined region between graphene sheets. Reported studies [14-16] suggest that the metal atoms make a sheathing organization over the graphene substrate during the processing of nano-composite systems. The utmost interface area between the metal - matrix and graphene substrate enhance the strength of the metal-matrix graphene composite against during external load applications. Hwang et al. [14] depicted the reduced graphene oxide homogeneously dispersed in the copper matrix, which acquired considerably improved mechanical performance by a molecular-level mixing method. Li et al. [15] have found that the Ni- coated graphene substrate not only prohibited the aggregation, but also improved the interface strength between graphene and the copper matrix. Salam et al. [17], have synthesized silver-graphene nanocomposites by experimental methodology. They have shown that the developed silver graphene nano-composite material has excellent catalytic properties, is inexpensive and can be reused five times without decrease in activity and selectivity. Bhunia and Jana [18] have developed a simple and a large-scale experimental methodology for the synthesis of graphene oxide-silver composite. They have found that the photo catalytic efficiency of graphene oxide-silver nano-composite under visible light is significantly higher. However, some experimental investigations have been reported that the inclusion of graphene in metals matrix decreases its mechanical properties due to poor graphene/metal interface adhesion/bonding and high processing temperatures [19-22].

Despite of a large number of reported experimental studies concerning to metal matrix nanocomposite, there are only a few studies are available using molecular dynamic simulations of metal/graphene nanocomposites [23,24]. Amal et al. [23] have investigated the distribution and evolution of atomic nanoclusters of variety of metallic materials over the graphene substrate using molecular dynamics

\footnotetext{
* Corresponding author.

E-mail address: sunil@nmlindia.org (S. Kumar).
} 


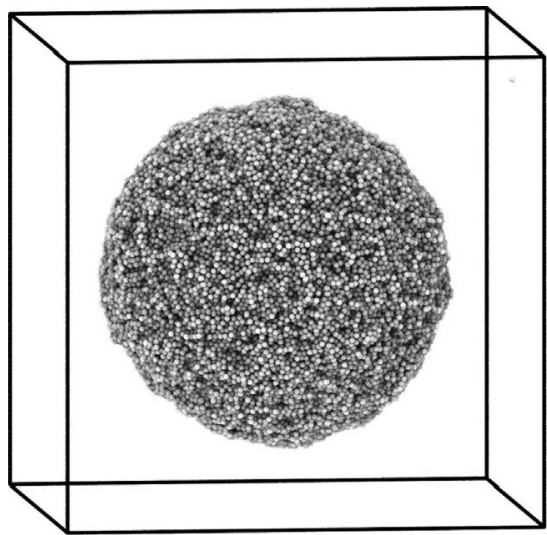

(a)

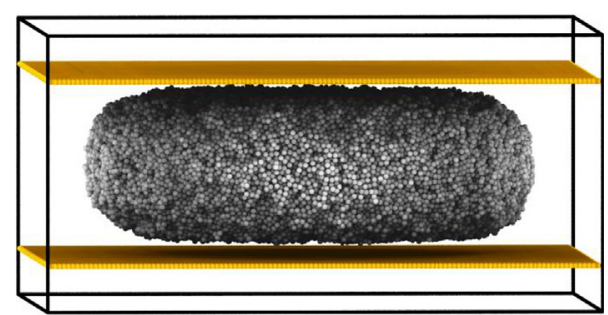

(b)
Fig. 1. Snapshots of simulation systems for solidification and structural organization of silver in bulk and in confined region: (a) silver (150,000 atoms) placed in a cubical simulation box of dimension ( $\pm 175 \AA$ \& $\pm 175 \AA, \pm 175 \AA$ ) and (b) silver (150000 atoms) placed in a confined region between two square graphene sheets (side length $350 \AA$ ). Graphene sheets have been placed along $x$-y plane and at $\mathrm{z}=0 \AA$ and $\mathrm{z}=100 \AA$.
Table 1

Embedded Atoms Method (EAM) due to Finnis-Sinclair (FS) [28] parameters for silver atoms.

\begin{tabular}{lllll}
\hline $\mathrm{m}$ & $\mathrm{n}$ & $\varepsilon(\mathrm{eV})$ & $\mathrm{c}$ & $\mathrm{a}(\AA)$ \\
\hline 6 & 12 & 0.025415 & 144.41 & 4.09 \\
\hline
\end{tabular}

simulations. They have found that the silver, gold, and copper formed aggregates or globules of different sizes, which was arbitrarily spread over the graphene substrate. On the contrary, lithium, sodium, and potassium have formed a mono-atomic layer distribution over the graphene substrate. Duan et al. [24] have developed a copper/graphene nanocomposites and investigated it's mechanical properties using molecular dynamics simulation. They found that the fracture strain, Young's modulus and the tensile strength of nanocomposites rises with increase in the number of graphene layers within the copper matrix. Despite these studies, the solidification, structural organization (phase transitions) and mechanical properties of metals in the confined region between graphene sheets still needs to be explored. To the best of our knowledge, the solidification, structural organization (phase transitions) and mechanical properties of metals in the confined region between graphene sheets by molecular dynamics simulation has not been studied till now.

In this study, the solidification, structural organization and its mechanical properties of silver nano-particle in a confined region between graphene sheets have been explored by molecular dynamics simulations. Silver is selected as a metal nanoparticle due to its wide applications in flexible electronics, biomedical devices, catalyst, etc. In the first step, adsorption and organization of silver nano-particle in a confined region between two graphene substrate was simulated. In the second step, the tensile deformation of composite system has been carried out at various temperatures to study the mechanical behaviour. The adaptive common neighbour analysis, radial distribution and potential energy evolution have been used to characterize the organization of silver nano-particle in the confined region.

\section{Simulation details}

Molecular dynamics simulation [25-27] has been carried out under the constant- number of atoms, volume and temperature (NVT) ensembles to study the solidification, structural organization and mechanical properties of silver nano-particle in bulk and the confined region between graphene sheets as shown in Fig. 1.To execute intraatomic interactions for silver nano-particle, Finnis and Sinclair[28] form of embedded atom method (EAM) potentials has been employed during molecular dynamics simulation. The total intra-atomic interaction potential energy of silver nano-particle, $E_{A g-A g}$ is estimated by

$E_{A g-A g}=\sum_{i} \varepsilon\left[\frac{1}{2} \sum_{j \neq i} \mathrm{~V}\left(r_{i j}\right)-c \sqrt{\rho_{i}}\right]$

$V\left(r_{i j}\right)=\left(\frac{a}{r_{i j}}\right)^{n}$

$\rho_{i}=\sum_{j \neq i}\left(\frac{a}{r_{i j}}\right)^{m}$

where $r_{i j}, a, c, n, m$, and $\varepsilon$ are depicting the separation between $i^{t h}$ and $j^{\text {th }}$ silver atom, lattice parameter and other positive constants for silver nano-particle respectively. $V\left(r_{i j}\right)$ is showing the pair intra-atomic potential energy to account for silver atom, and $\rho_{i}$ the local charge density. Eqs. (1)-(3) can be written in the generalized form of Hamiltonian pertaining to silver atoms as given by equation (4) below

$H=\frac{1}{2}\left[\sum_{i \neq j} \sum \hat{p}_{i} \hat{p}_{j} V\left(r_{i j}\right)\right]-d \sum_{i} \hat{p}_{i}\left[\sum_{j \neq i} \hat{p}_{j} \varnothing\left(r_{i j}\right)\right]^{1 / 2}$

$\hat{p}_{i}=\left\{\begin{array}{lc}1, & \text { if site } i \text { is occupied by a metal atom, } \\ 0, & \text { otherwise }\end{array}\right.$

$V(r)=\varepsilon\left[\frac{a}{r}\right]^{n}$

$\varnothing(r)=\varepsilon\left[\frac{a}{r}\right]^{m}$

$d=\varepsilon \times c$

The numerical values of $\varepsilon, a, c, m$ and $n$ for the silver atoms have been given in Table 1. The Finnis and Sinclair form of embedded atom method potential is a semi-empirical method for performing molecular dynamics simulation of metallic materials. However, it has some limitations, like no angular dependency on electron density, without consideration of covalency of metal atoms, and excluding Fermi-surface effects [47]. Despite of these limitations, Finnis and Sinclair form of embedded atom method potentials are widely incorporated during 


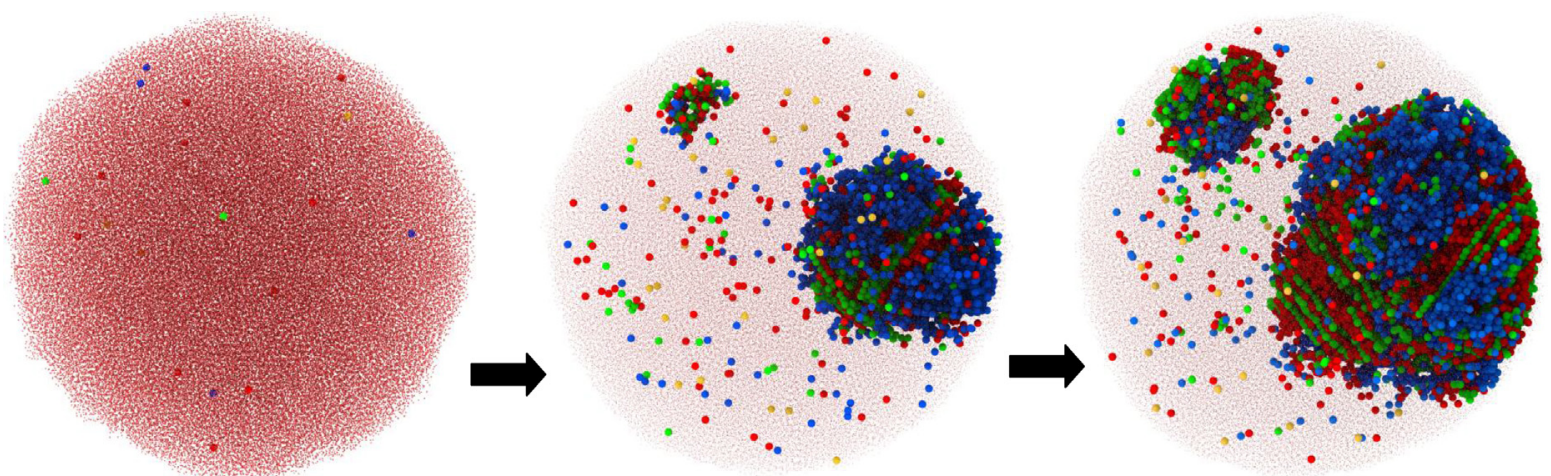

$1500 \mathrm{~K}$

$1025 \mathrm{~K}$
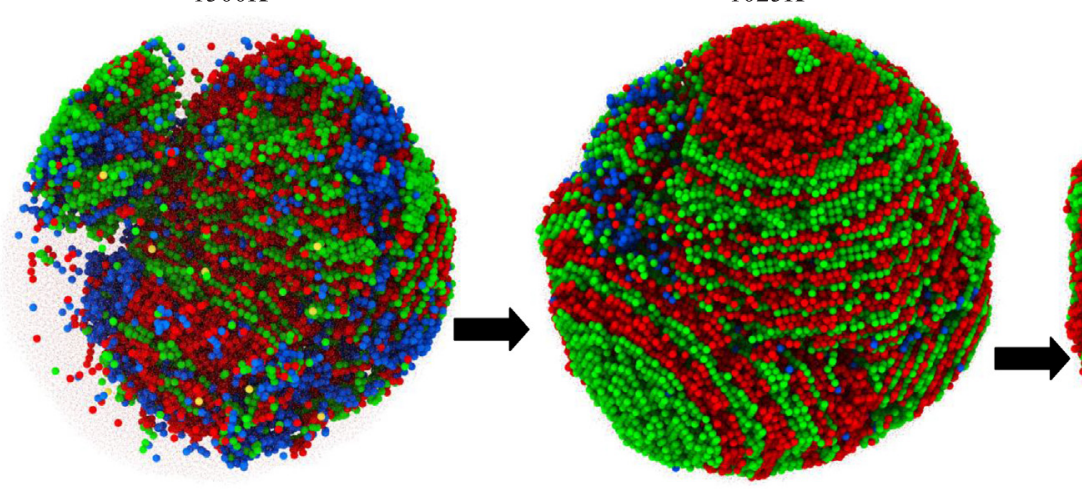

$1020 \mathrm{~K}$

$1010 \mathrm{~K}$

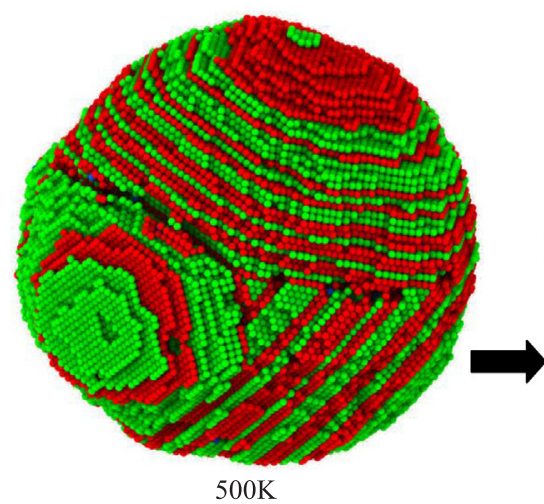

$1000 \mathrm{~K}$

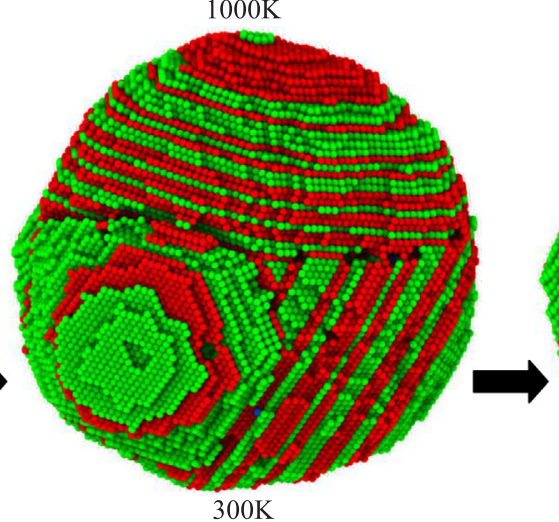

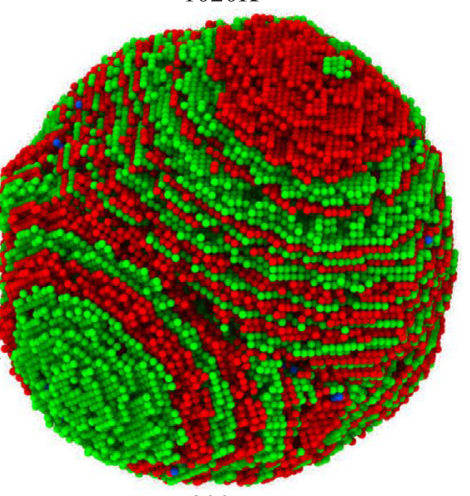

$900 \mathrm{~K}$

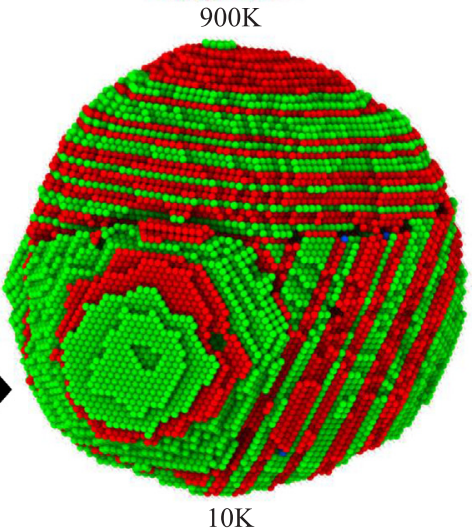

$10 \mathrm{~K}$

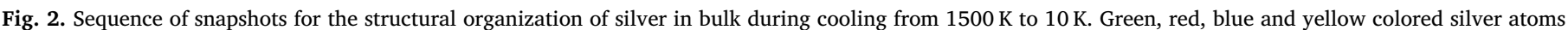

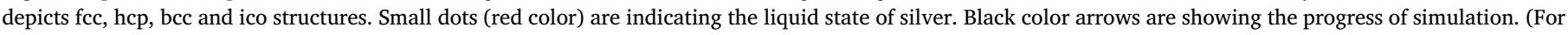
interpretation of the references to color in this figure legend, the reader is referred to the web version of this article.)

molecular dynamics simulations of metallic materials. It provides reasonably precise results at a nominal computational expense, from which many desirable properties of metal can be predicted at atomic length scale. Crystallization process, sequence of crystalline structures, cohesive energy of silver form molecular dynamics simulations have been found nearly accurate as reported results in the Refs. [48-49].

The inter-atomic potential energy between silver $(\mathrm{Ag})$ and carbon (C) atoms of graphene sheets have been implemented through 12-6 Lennard Jones (LJ) potential energy as given in the following equation (9).

$E_{L J}=4 \varepsilon_{A g-C}\left[\left(\frac{\sigma_{A g-C}}{r}\right)^{12}-\left(\frac{\sigma_{A g-C}}{r}\right)^{6}\right]$

where $\varepsilon_{A g-C}$, and $\sigma_{A g-C}$ are known as 12-6 LJ parameters for the energy and equilibrium inter-atomic distance respectively. The values of $\varepsilon_{A g-C}=0.0301 \mathrm{eV}$ and $\sigma_{A g-C}=3.006 \AA$ have been used for the silvergraphene (Ag-C) nano-composite system as given in Ref. [29]. The Newton's equation of motion of each silver atom due to the both interand intra-atomic forces arising from various interaction potentials can be carried out as given in equation (10)

$w \times \frac{d^{2} \boldsymbol{r}_{i}}{d t^{2}}=\boldsymbol{F}_{i}=-\nabla_{i} E$

where $\boldsymbol{F}_{i}$ represent the total force acting on the $i^{\text {th }}$ silver atom by other surrounding atoms. The Newtonian equation of motion for silver atoms have been solved by velocity Verlet algorithm [30] with a time step $(\Delta t)$ of 2 femto-seconds. The Nose-Hoover thermostat [31,32] has been used to control the variations in temperature of the silver nano-particle 


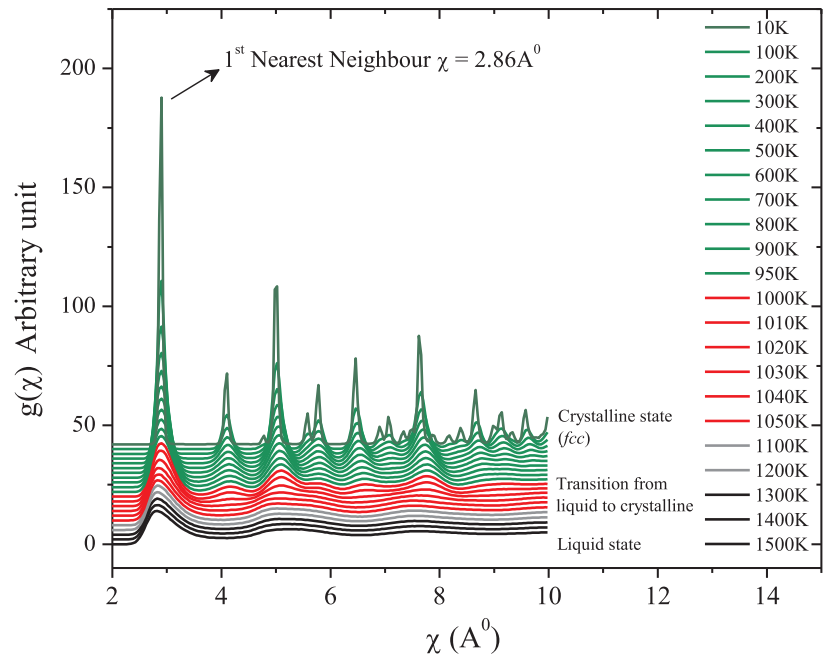

Fig. 3. Radial distribution function, $g(\chi)$, during solidification and structural organization of silver in bulk during cooling from 1500 to $10 \mathrm{~K}$. Liquid, transition from liquid to crystalline and crystalline states are shown by black, red, and green color curve lines at respective temperatures. (For interpretation of the references to color in this figure legend, the reader is referred to the web version of this article.)

during simulations. Molecular dynamics simulation of silver nano-particle graphene nano-composite has been carried out by Large Scale Atomic/Molecular Massively Parallel Simulator (LAMMPS) [33] open source software. Visualization and analysis of simulation data and trajectory have been carried out by Open Visualization Tool [34,35] and Visual Molecular Dynamics [36] software packages.

\subsection{Simulation system and processing steps}

We have considered two sets of the simulation systems. In the first system, silver nano-particle considered in bulk and in the second system, silver nano-particle placed in a confined region between graphene sheets, as shown in Fig. 1(a) and (b). An equal number of silver atoms, $\mathrm{N}_{\mathrm{Ag}}=150,000$, have been taken for both systems. Square graphene sheets (side length $350 \AA$ ) placed along the $x-y$ plane (at $z=0$ and $\mathrm{z}=100 \AA$ ) in a 3-D simulation box. In the first step, silver is melted at a temperature $(\mathrm{T}=1500 \mathrm{~K})$ for 1000 pico-second, to achieve an equilibrium and homogeneous liquid state of silver. The melting temperature of bulk silver nano-particle is $1234 \mathrm{~K}$, observed using experimental methods as reported by Chen et al. [37]. A moderately high temperature, $1500 \mathrm{~K}$, was selected for melting of silver nano-particle to reach into a homogeneous liquid state. In the second step, silver nanoparticle has been placed in a confined region between two graphene sheets at a temperature $1500 \mathrm{~K}$. In the third step, the simulation systems have been solidified by decreasing the temperature from $1500 \mathrm{~K}$ to $10 \mathrm{~K}$ (cooling rate of $0.1 \mathrm{~K} / \mathrm{ps}$ ). The graphene sheet has been abled to withstand temperature up to $3100 \mathrm{~K}$ (as estimated by molecular dynamics simulations [38]) and $2073 \mathrm{~K}$ (as estimated using experimentally [39]).

\subsection{Radial distribution function $g(\chi)$}

The radial distribution function [40] $g(\chi)$ of silver atoms in bulk and confined region has been defined as given in equation (11)
$g(\chi)=\frac{V}{N_{A g}^{2}}\left\langle\sum_{i=1}^{N_{A g}} \frac{n(\chi)}{4 \pi \chi^{2} \Delta \chi}\right\rangle$

where $N_{A g}$ and $V$ are the total number of silver atoms and volume of the 3-D simulation system respectively. Further, $n(\chi)$ has been depicted the number of silver atoms reside in a spherical shield of radius $\chi$ and thickness $\Delta \chi$ from the central atom.

\subsection{Adaptive common neighbor analysis}

The adaptive Common Neighbour Analysis (a-CNA) [34,35] is applied to illustrate the organization of silver atoms in the bulk as well as in a confined region between graphene sheets. The $a-C N A$ is capable for the recognizing the silver atoms belongs to a multiplicity of crystal structure types such as face centered cubic $(f c c)$, body centered cubic $(b c c)$, hexagonal closed packed ( $h c p$ ), icosahedra (ico) and others. In the a-CNA, the multiplicity of the crystalline structure has been recognized by the guess of three integers $\left(n_{\mathrm{cn}}, n_{\mathrm{b}}\right.$, and $\left.n_{\text {lcb }}\right)$ for each central silver atom [41-44]. $n_{\mathrm{cn}}$ is the number of common neighbour of silver atom, $n_{\mathrm{b}}$ is the number of bonds between $n_{\mathrm{cn}}$ common neighbours. $n_{\text {lcb }}$ is the number of bonds in the highest continuous chain produced by the $n_{\mathrm{b}}$ bonds between common neighbour of silver atoms. For the $f c c$ structure of silver atoms, the integers are of the kind $\left(n_{\mathrm{cn}}, n_{\mathrm{b}}, n_{\mathrm{lcb}}\right)=(4,2,1)$ or simply known as 421 . For the $h c p$ structure of silver atoms, $50 \%$ integers are of kind 422 and another $50 \%$ integers are of kind 421. The $b c c$ structure of silver atoms consists of both 441 and 661 kinds.

\subsection{Average number distributions}

The distributions of silver atoms, $\frac{n(z)}{N_{A g}}$, in the confined region between graphene sheets have been calculated with the z-direction; $n(z)$ is the number of silver atoms reside in a planner shell of thickness, $\Delta \mathrm{z}$; $N_{A g}=150,000$, is the total number of silver atoms present in the confined region between graphene sheets.

\section{Results and discussion}

The results obtained from molecular dynamics simulation for solidification, structural organization, and mechanical properties of silver nano-particle in both bulk and confined region has been discussed in the next three subsections. In the first sub-section, solidification and structural organization of silver in bulk will be discussed. In the second sub-section, we have described the solidification, adsorption and structural organization of silver nano-particle in the confined region between two graphene sheets. Mechanical properties of silver nanoparticle by tensile deformation has been estimated in the third subsection.

\subsection{Solidification and structural organization of silver nano-particle in the bulk}

The Solidification and structural organization process of silver nanoparticle in the bulk have studied by cooling from $1500 \mathrm{~K}$ to $10 \mathrm{~K}$ (cooling rate $=0.1 \mathrm{~K} / \mathrm{ps}$ ). The sequence of snapshots for solidification and structural organization of silver have been depicted at various temperatures as shown in Fig. 2. Silver starts to organize in $f c c, h c p, b c c$ and ico structures at a temperature $\mathrm{T}=1025 \pm 25 \mathrm{~K}$ during cooling process from $1500 \mathrm{~K}$ to $10 \mathrm{~K}$. At the early stage of the nucleation (at 


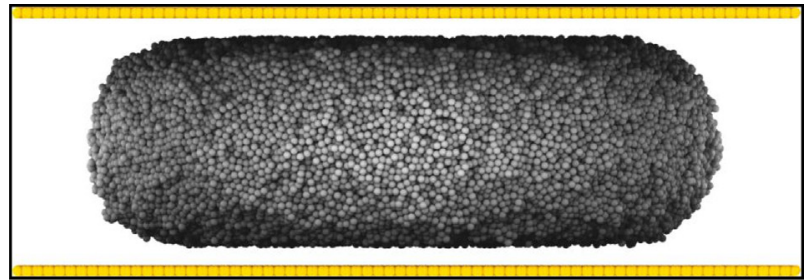

Initial position of silver in confined region between graphene sheets

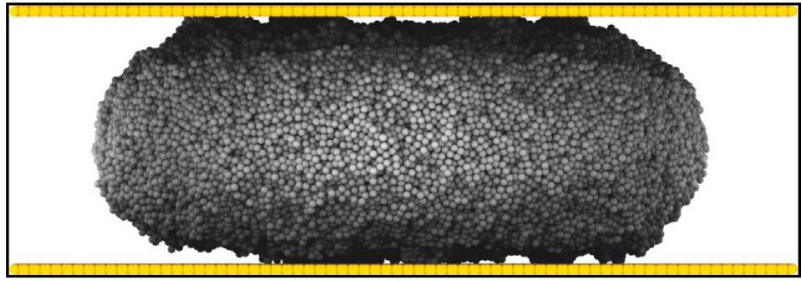

$30 \mathrm{ps}$

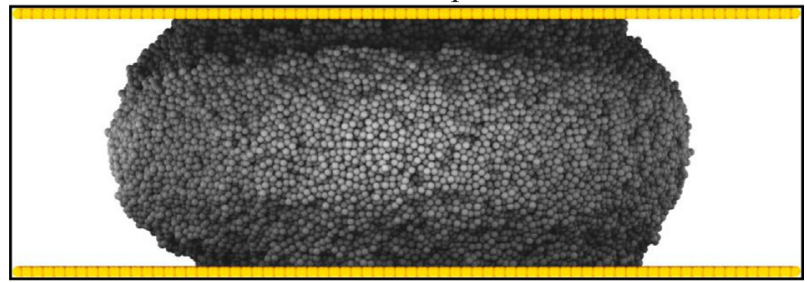

$50 \mathrm{ps}$

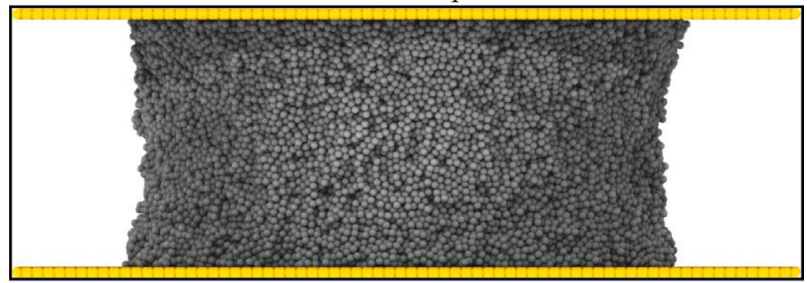

$500 \mathrm{ps}$

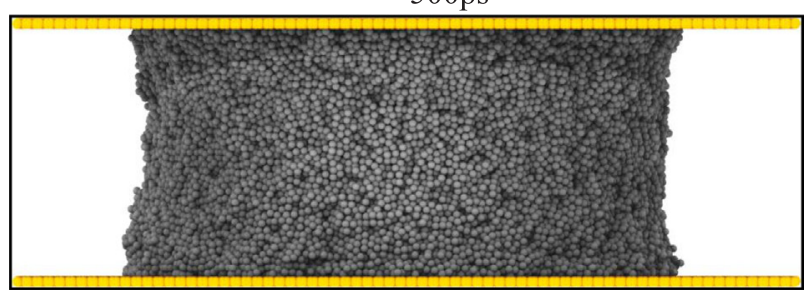

$1000 \mathrm{ps}$
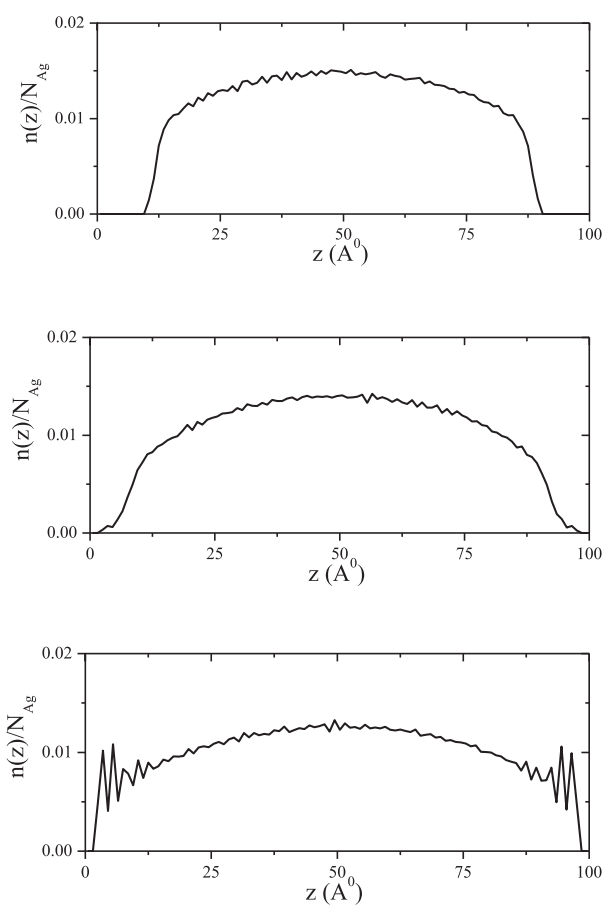

$\downarrow$
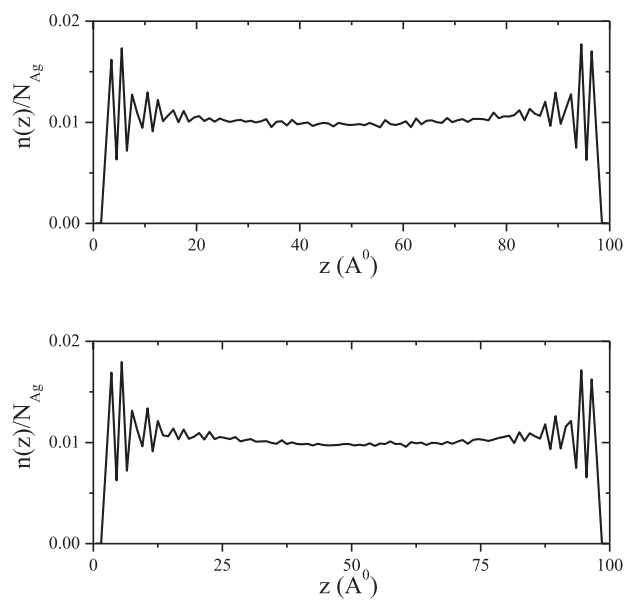

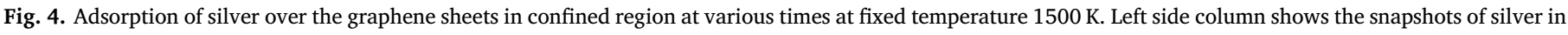

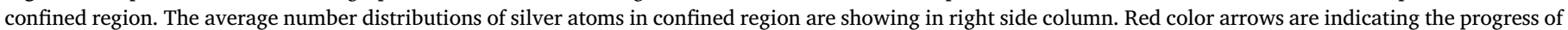

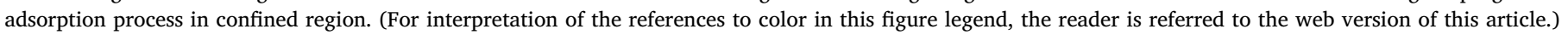

$\mathrm{T}=1025 \mathrm{~K}$ to $1010 \mathrm{~K}$ ), silver atoms are organized into $b c c$ structure. Further, as temperature decreases below $\mathrm{T}=1010 \mathrm{~K}$, silver atoms from $b c c$ structures are transformed into $f c c$. These observations are according to the Ostwald's step rule, in which a metastable bcc evolves from liquid and then transform into a stable $f c c$ crystals [51]. It is due to this $b c c$ crystal depict comparatively higher energy and volume compared to $f c c$ structure. The radial distribution function, $g(\chi)$, has been plotted for silver atoms at various instants as temperature decreases from $1500 \mathrm{~K}$ to $10 \mathrm{~K}$ as shown in Fig. 3. At high temperatures i.e. $1500 \mathrm{~K}$ to $1050 \mathrm{~K}$, distinct peaks of $g(\chi)$ are evolved at $\chi=2.86 \AA$, due to a short range ordered structures of silver atoms in the liquid state. These peaks depict to the first nearest neighbours (1st N.N.) of silver atoms in bulk, which is found to be equal to $a \times \frac{\sqrt{2}}{2}=2.89 \AA$, where $a$ is the lattice parameter for silver crystal $(a=4.09 \AA)$. Further, in the temperature range $1500 \mathrm{~K}$ to $1050 \mathrm{~K}$, no any distinct and identifiable peak has found at $\chi>2.86 \AA$, which may signify to the lack of long range ordered correlations. At temperatures below $\mathrm{T}=1010 \mathrm{~K}$, the many peaks of $g(\chi)$ vs $\chi$ curve are evolve gradually due to both short and long range structural evolutions among in the silver atoms. 


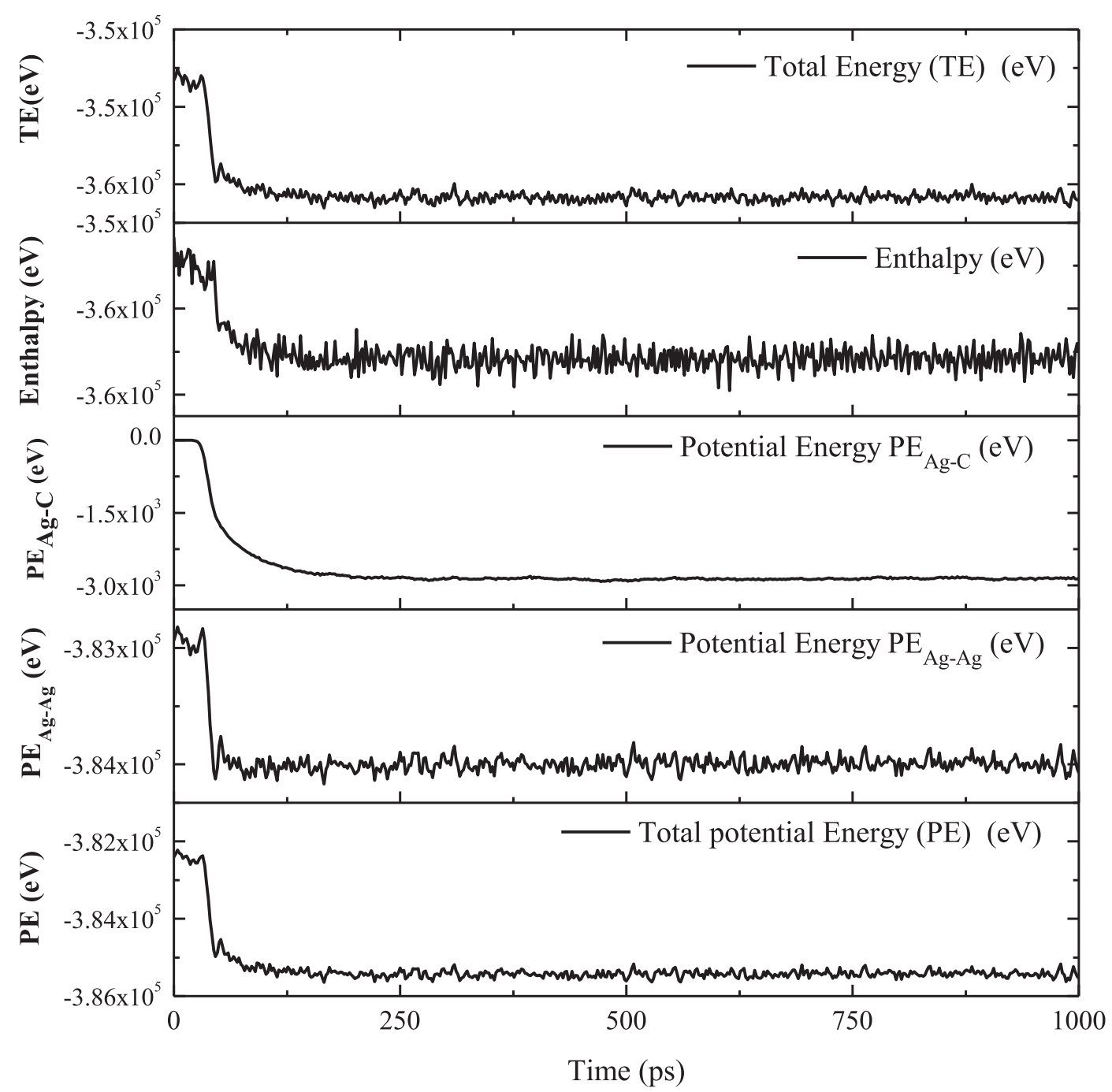

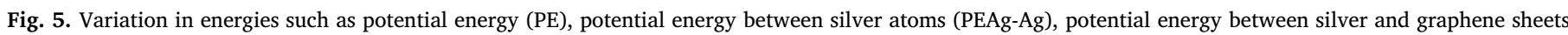
(PEAg-C), enthalpy and total energy (TE) during adsorption of silver in confined region between over two graphene sheets at $1500 \mathrm{~K}$.

3.2. Adsorption, solidification and organization of silver nano-particle in the confined region between graphene sheets

Snapshots for the adsorption process of silver nano-particle in a confined region between two graphene sheets at various time (at fix temperature $\mathrm{T}=1500 \mathrm{~K}$ ) have shown in Fig. 4. It was found that the silver nano-particle gradually adsorbs over the graphene sheets in the confined region and reached in equilibrium within 500 ps as shown by snapshot and average number distributions. The average number distribution of silver atoms, $\frac{n(z)}{N_{A g}}$, in a confined region has been calculated as given in right side column of Fig. 4. As simulation proceed, silver nano-particle gradually migrates toward the graphene sheets in the confined region and adsorb over it, the corresponding average number distribution of silver atom shift toward the $\mathrm{z}=0 \AA$ and $\mathrm{z}=100 \AA$ due to adsorption and spreading of the silver atom over the graphene sheets.

Fig. 5 shows the variations in different energies such as potential energy ( $\mathrm{PE})$, potential energy between silver atoms $\left(\mathrm{PE}_{\mathrm{Ag}-\mathrm{Ag}}\right)$, nonbonded interaction potential energy between silver and graphene sheets $\left(\mathrm{PE}_{\mathrm{Ag}-\mathrm{C}}\right)$, enthalpy and total energy (TE) during adsorption of silver nano-particle in a the confined region between two graphene sheets at $1500 \mathrm{~K}$. During the adsorption process in confined region, potential energy $\left(\mathrm{PE}_{\mathrm{Ag}-\mathrm{C}}\right)$ decreases due to the spreading of silver nano-particle over the graphene substrate. Furthermore, silver atoms are re-organized in a confined region to minimize inter-atomic potential energy $\left(\mathrm{PE}_{\mathrm{Ag}}\right.$ $\mathrm{Ag}$ ), total energy (TE) and enthalpy. It is expected that $\mathrm{PE}_{\mathrm{Ag}-\mathrm{C}}$ is the driving potential energy for the adsorption of metallic materials over the graphene or carbon nano-tube substrate $[45,46]$. Fig. 6 shows the snapshots of structural evolutions in silver nano-particle in the confined region between two graphene sheets during solidification from $1500 \mathrm{~K}$ to $10 \mathrm{~K}$. At high temperature, $\mathrm{T}>1270 \pm 20 \mathrm{~K}$, silver nano-particle depicted a random organization (liquid phase) in the confined region. Further, as temperature decreased below $\mathrm{T}<1270 \mathrm{~K}$, the silver atoms start to organize in various structures such as $f c c, b c c, h c p$, ico etc. The 


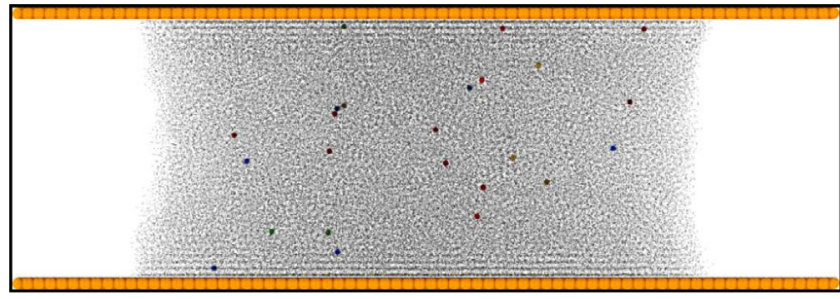

$1500 \mathrm{~K}$
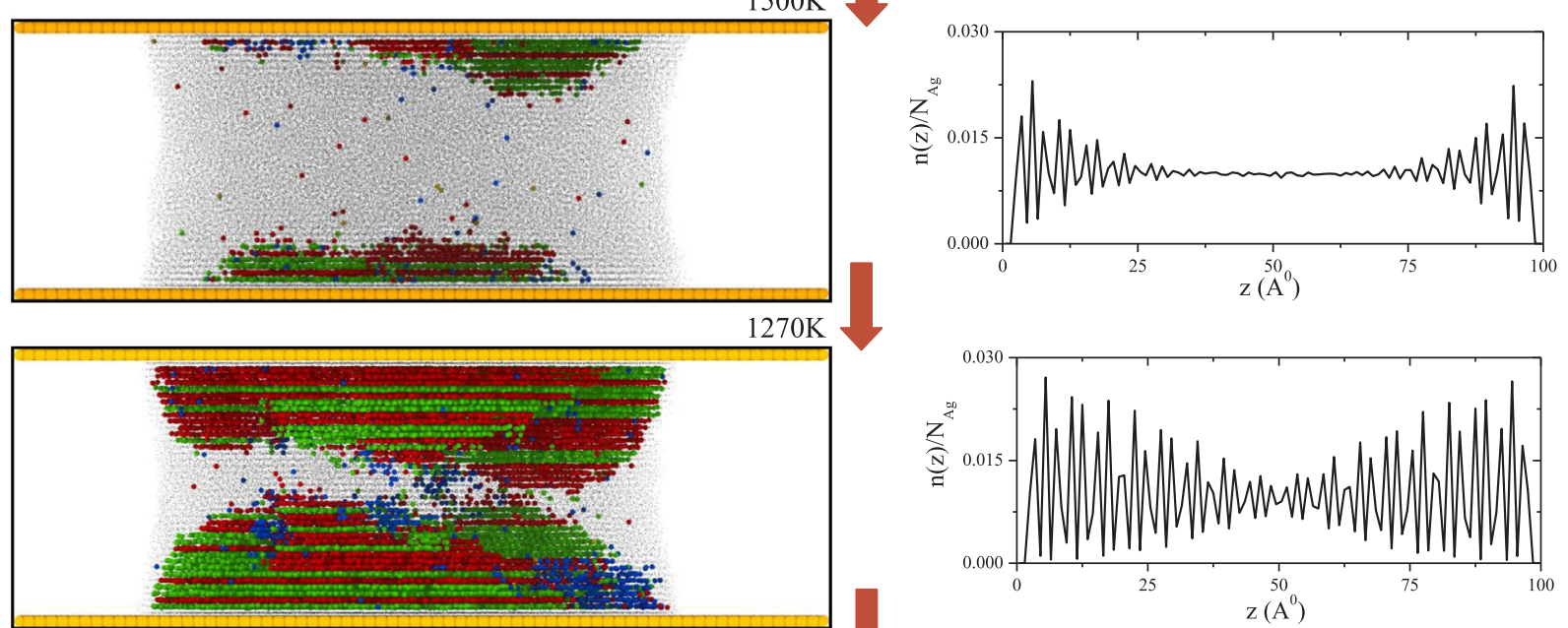

$1260 \mathrm{~K}$

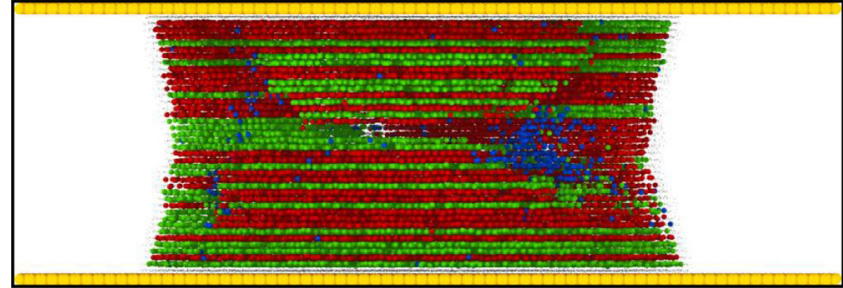

$1250 \mathrm{~K}$

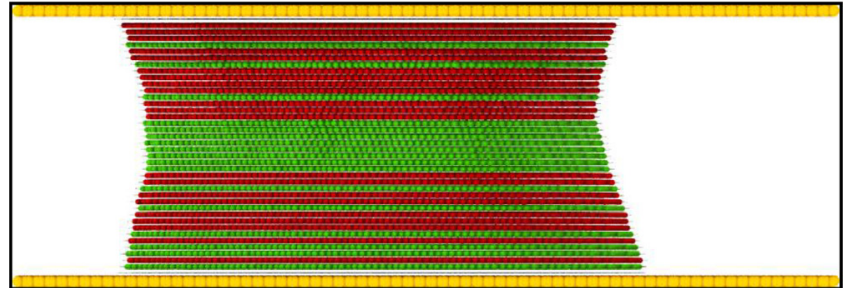

$300 \mathrm{~K}$
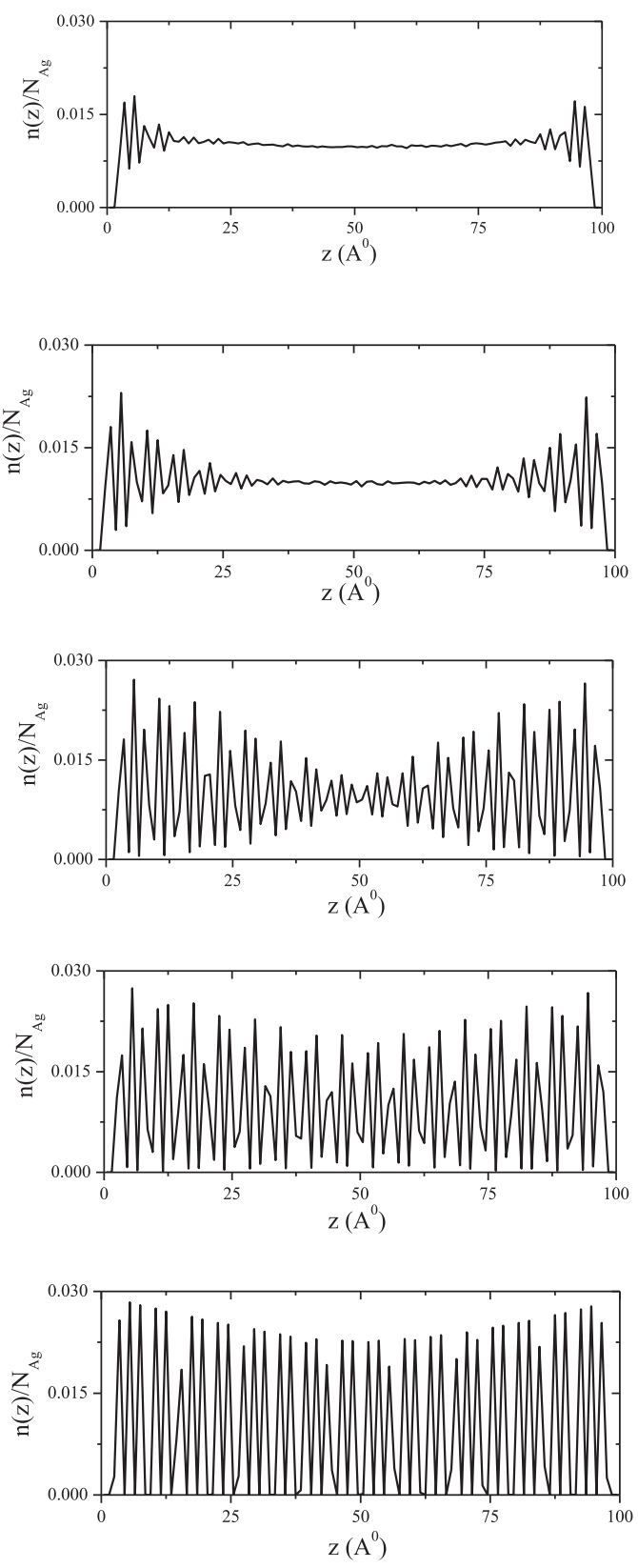

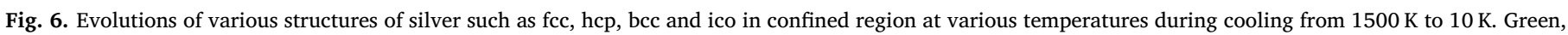

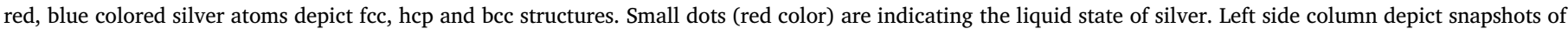

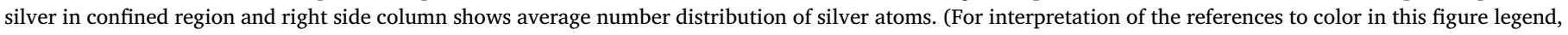
the reader is referred to the web version of this article.)

structural organization of silver nano-particle in a confined region has observed to facilitate by graphene sheets. Therefore, initiation and progress of crystallization occur near to the graphene sheets. Finally, at temperature $300 \mathrm{~K}$, silver nano-particle organized in $f c c$ structures with layered $h c p$ organization in the confined region.

In general, silver nano-particle depicts poor wettabilty with the carbonaceous nano-materials such as graphene, carbon nanotubes, fullerene (bucky balls) etc. Poor wetting generally leads to weak interfacial adhesion and aggregation of nano-materials in silver matrix nanocomposites. The extent of wetting of silver nano-particle with graphene sheets can be modelled by tuning the 12-6 LJ potential energy parameter $\left(\varepsilon_{\mathrm{Ag}-\mathrm{C}}\right)$ for understanding the phenomena at the atomic

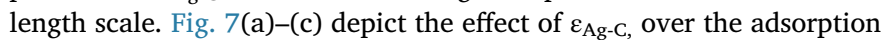
and structural organization of silver nano-particle in a confined region 


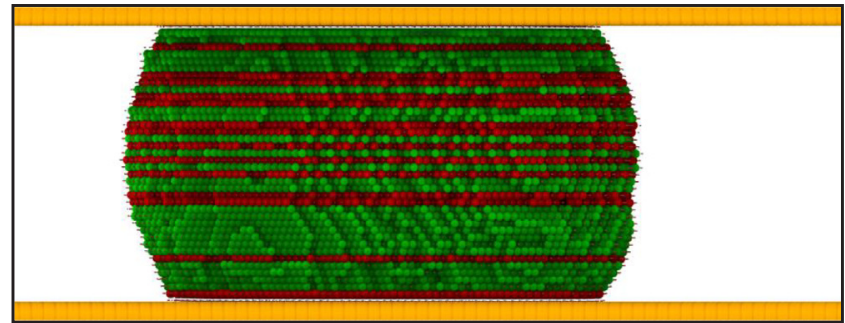

(a)

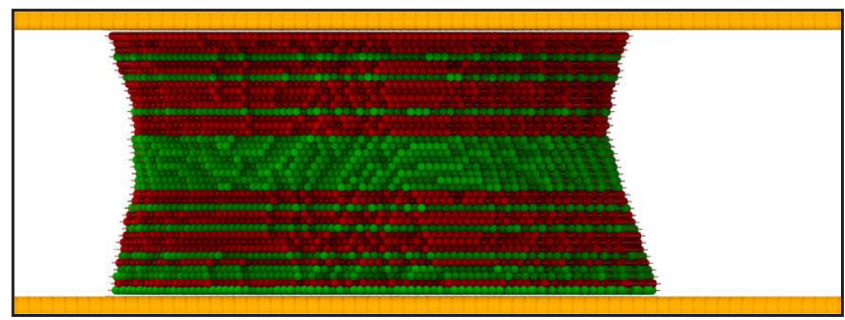

(b)

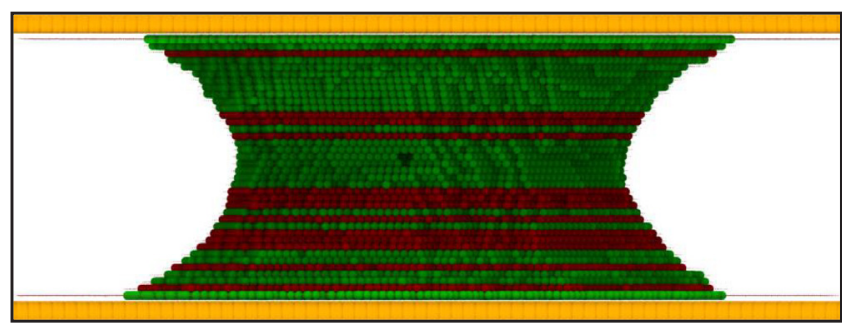

(c)
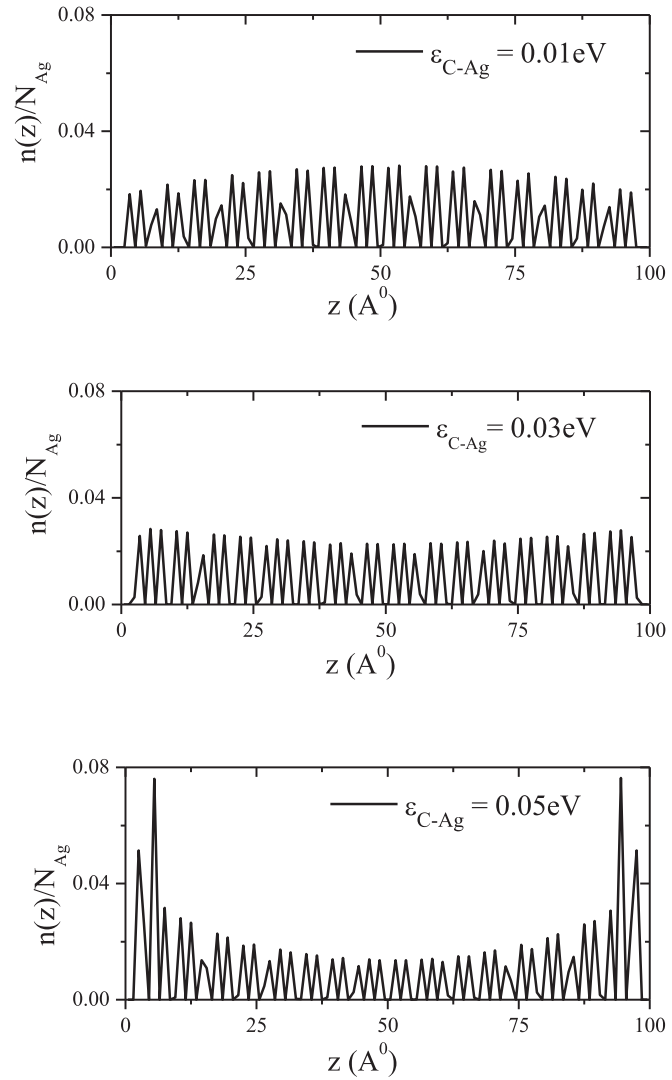

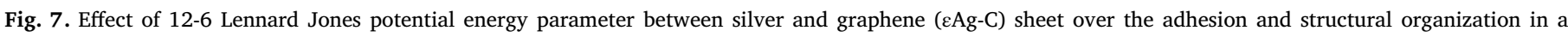

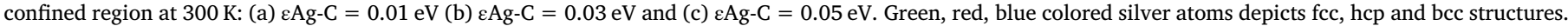

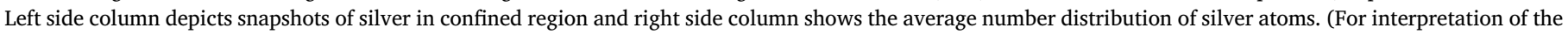
references to color in this figure legend, the reader is referred to the web version of this article.)

between graphene sheets. At the lowest value of $\varepsilon_{\mathrm{Ag}-\mathrm{C}}=0.01 \mathrm{eV}$, the silver nano-particle show very poor wetting over the graphene sheets and make a globule like structure in the confined region. With the increasing value of $\varepsilon_{\mathrm{Ag}-\mathrm{C}}$, silver nano-particle preferred to adsorbed over both graphene sheets as shown in Fig. 7(b) and (c). This indicates that the $\varepsilon_{\mathrm{Ag}-\mathrm{C}}$ controls the compatibility and wettabilty between graphene and silver in a confined region. Higher values of $\varepsilon_{\mathrm{Ag}-\mathrm{C} \text {, designate strong }}$ adhesion or high wettabilty between the graphene and silver. Average number distributions $\left(\frac{n(z)}{N_{A g}}\right)$ of silver in the confined region have plotted in right-side column of Fig. 7. The multiple peaks of $\frac{n(z)}{N_{A g}}$ vs z curve depicts parallel layered organization of silver in the confined region. At low values of $\varepsilon_{\mathrm{Ag}-\mathrm{C}}=0.01 \mathrm{eV}$, the values of $\frac{n(z)}{N_{A g}}$ found lower at near the graphene sheets (i.e. at $\mathrm{z}<10 \AA$ and $\mathrm{z}>90 \AA$ ) compared to middle of the confined region (i.e. at $10 \AA<\mathrm{z}<90 \AA$ ), which indicates the poor wettabilty of silver nano-particle over both the graphene sheets. On the contrary, at relatively higher values of $\varepsilon_{\mathrm{Ag}-\mathrm{C}}=0.03 \mathrm{eV}$ and $0.05 \mathrm{eV}$, the values of $\frac{n(z)}{N_{A g}}$ found higher at near the graphene sheets compared to middle of confined region, which indicates the good wettabilty or strong adhesion with the graphene sheets.

Fig. 8 (a) and (b) depicts the variation in total energy and percentage of crystalline atoms of silver nano-particle in bulk and the confined region between two graphene sheets at various values of $\varepsilon_{\mathrm{Ag}-\mathrm{C}},(0.01 \mathrm{eV}$,
$0.03 \mathrm{eVand} 0.05 \mathrm{eV}$ ) during cooling from 1500 to $10 \mathrm{~K}$. Total energy of silver nano-particle in both, bulk and confined region gradually decreases with a decrease in temperature. The total energy of silver nanoparticle in bulk depict a sharp decrease at a temperature of $1030 \mathrm{~K}$ due to phase transition or evolution of various crystalline structure such as $f c c, h c p, b c c$, ico etc. However, the energy of silver nano-particle in the confined region with various values of $\varepsilon_{\mathrm{Ag}-\mathrm{C},}=0.01 \mathrm{eV}, 0.03 \mathrm{eV}$ and $0.05 \mathrm{eV}$, also depict sharp decrease in total energy at $1100 \mathrm{~K}, 1265 \mathrm{~K}$ and $1360 \mathrm{~K}$ due to the phase transition in a confined region. Similarly, the comparison of temperature for the evolution of the crystalline phase of silver in bulk and the confined region has plotted in Fig. 8(b). From total energy and percentage of crystallisation vs temperature variations, it can be concluded that phase transition of silver nano-particle from liquid to crystalline in the confined region between two graphene sheets have found to be dependent on the values of $\varepsilon_{\mathrm{Ag}-\mathrm{C}}$.

\subsection{Estimation of stress and dimension-less aspect ratio during tensile deformation}

For the estimation of the strength of adhesion between silver and graphene, we have pulled the graphene sheets along the $\pm \mathrm{z}$ direction by constant velocity $\left(\mathrm{v}_{\mathrm{z}}= \pm 1 \AA / \mathrm{ps}\right)$ at various temperatures during solidification as shown in Figs. 9-11. At $300 \mathrm{~K}$, the detachment occur at the interface between silver and graphene at a very early stage of tensile 


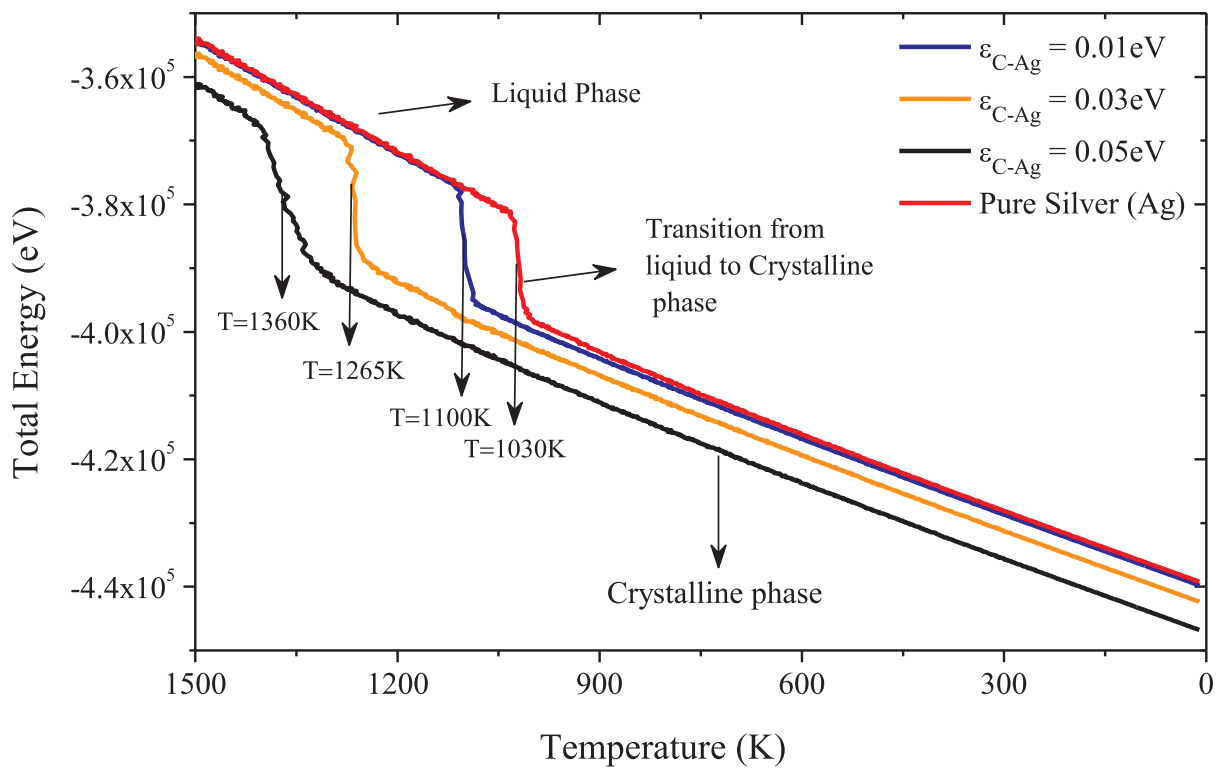

(a)

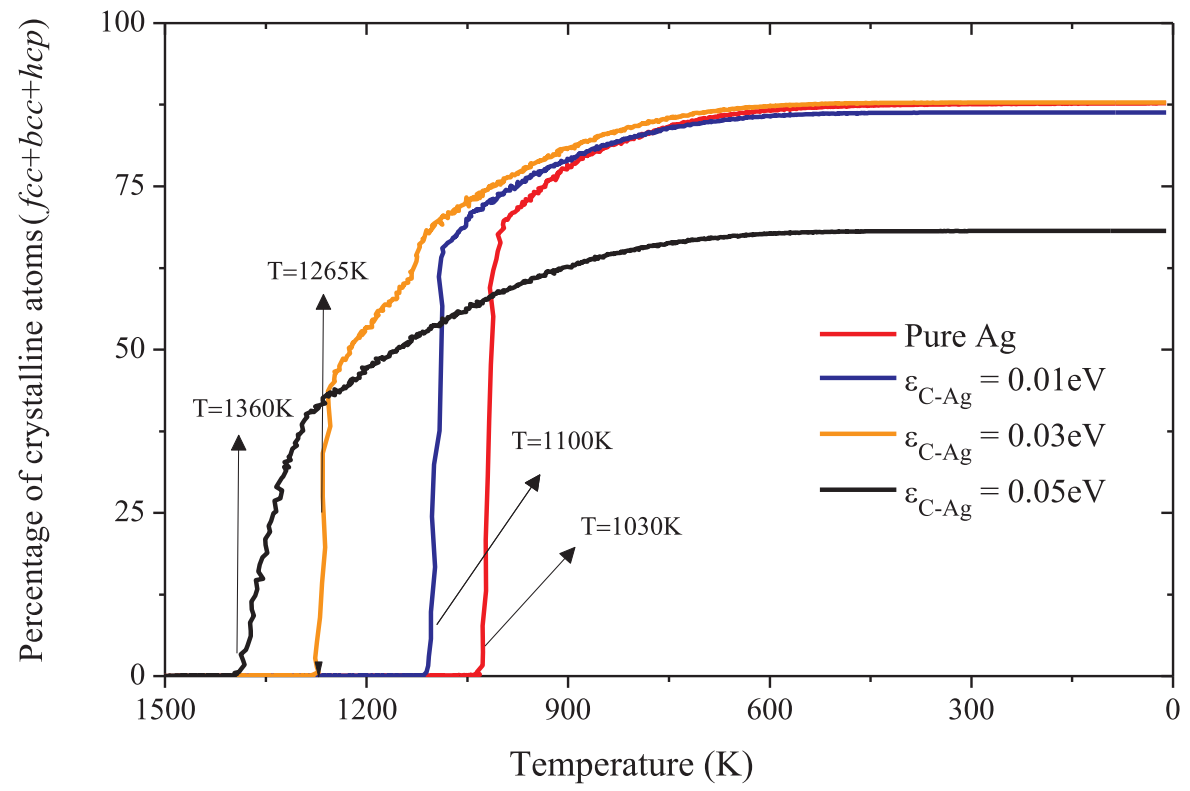

(b)

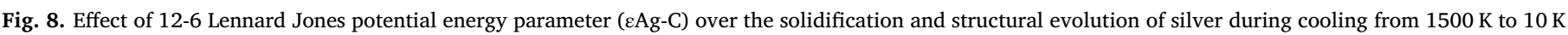
both in bulk and confined region: (a) variation in total energy and (b) percentage of crystalline atoms (fcc + hcp + bcc + ico).

deformations as shown in Fig. 9(a). The average number distribution of silver atoms, $\frac{n(z)}{N_{A g}}$, does not change during tensile deformation due to smooth detachment between silver and graphene sheet. At $1200 \mathrm{~K}$, the detachment has not observed at the interface, but it elongated during tensile deformation of silver. The average number distribution, $\frac{n(z)}{N_{A g}}$, has become broadened as tensile deformation proceeds as shown in Fig. 10. At $1500 \mathrm{~K}$, silver is in the liquid state and depict extensive elongation without detachment during tensile deformation as shown in Fig. 11. Such kind of tensile deformations of silver nano-cluster depicts enormous applications in the nanoelectronics, electrodes, catalysts, interconnects, antenna etc. in which crystal stability at various temperatures significantly influence the desirable property as reported by Sun et al.[50]

Further, we have investigated the evolution of solid volume, solvent accessible surface area (SASA), dimensionless aspect ratio and stress 


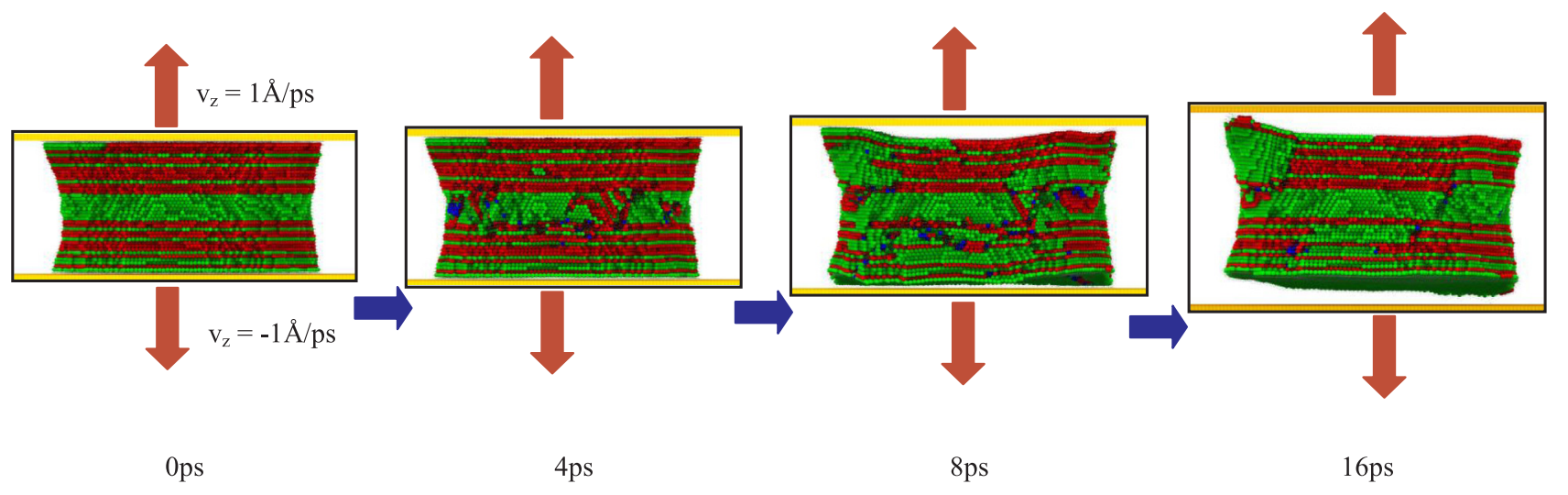

(a)
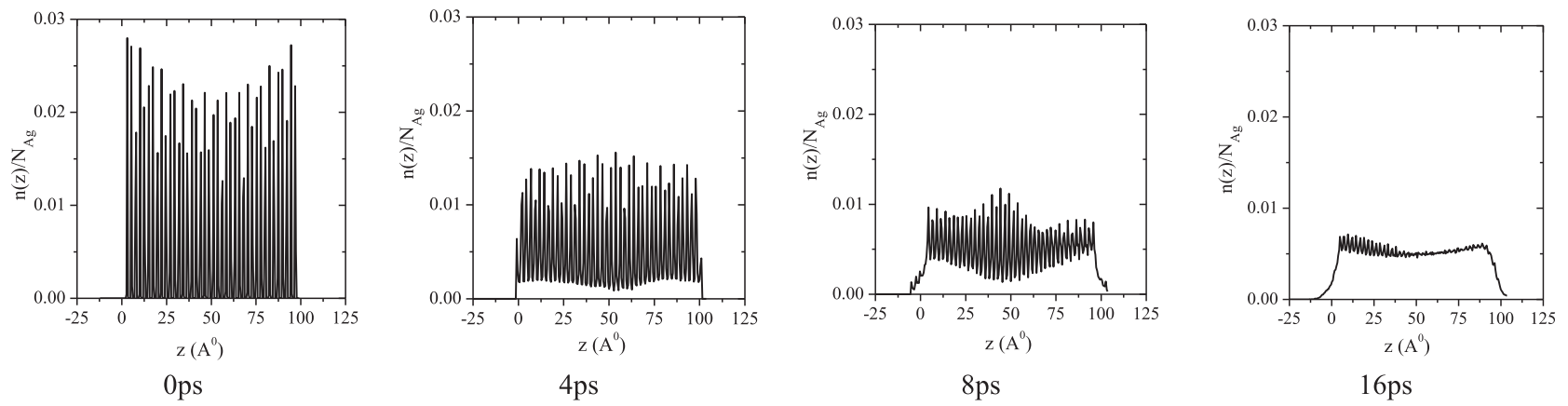

(b)

Fig. 9. Visuallization of detachment of silver in confined region between two graphene sheets during tensile pulling of graphene sheet by vz = $1 \AA / p s$ and $-1 \AA / p s$ at $300 \mathrm{~K}$ : (a) snapshot during tensile pulling and (b) average number distribution, $\frac{n(z)}{N_{A g}}$, of silver in a confined region at various instant.

during tensile deformation as shown in Fig. 12(a)-(d). Under tensile deformation, evolution of solid volume, $\Delta \mathrm{V}=\mathrm{V}(\mathrm{t}=\mathrm{t})-\mathrm{V}(\mathrm{t}=0)$, has depicted swelling to relaxation sequence. In case of swelling, volume of silver increases, but during the relaxation volume of silver decrease due to initiation of slipping between the atoms. The evolution of Solvent Accessible Surface Area (SASA), $\triangle \mathrm{SASA}=\mathrm{SASA}(\mathrm{t}=\mathrm{t})-\mathrm{SASA}(\mathrm{t}=0)$ for crystalline silver $(\mathrm{T}=300 \mathrm{~K}, 600 \mathrm{~K}, 900 \mathrm{~K}$ and $1200 \mathrm{~K})$ increase during early stage of tensile deformation, but it decrease after initiation of cracks as shown in Fig. 12 (b). The dimensionless aspect ratio $\kappa=\frac{\Delta S A S A^{3}}{\Delta V^{2}}$, is abled to characterize elongation of silver at various temperature during tensile deformation. The value of $\kappa$ was found to be stagnant during the early stage of tensile deformation, but it shows a sharp peak onset of detachment of silver from both graphene sheets as shown in Fig. 12(c). The stress values have been depicted at the various temperature $\mathrm{s}$ in Fig. 12(d). The yield stress was found to attain the maximum at $300 \mathrm{~K}$ due to the maximum interfacial adhesion. The effect of $\varepsilon_{\mathrm{Ag}-\mathrm{C}}$ over the detachment and elongation of silver during tensile deformation have shown in Fig. 13(a)-(c). It has been observed that detachment occurs at the interface for lower values of $\varepsilon_{\mathrm{Ag} \text {-C }}$ (for 0.01 and 0.03 ), on the contrary, at a high value of $\varepsilon_{\mathrm{Ag}-\mathrm{C}}=0.05 \mathrm{eV}$, detachment found within the silver nano-particle. These observation depict that the value of $\varepsilon_{\mathrm{Ag} \text {-C }}$ is abled to guide the detachment or evolution of cracks in the material during tensile deformations.

\section{Conclusion}

In this study, we have studied the solidification and various structural and as well as mechanical properties of silver nano-particle in a the confined region between graphene sheets by molecular dynamics simulations. The non-bonded interaction potential energy between silver atoms and graphene is one of the major energetic factor to control the structural organization of silver in the confined region. The major new finding for the solidification, structural organization and tensile deformations of silver (nano particle) in bulk and confined region are as given below:

- The phase transition from liquid to crystalline state of silver in the confined region observed relatively higher temperature compared to the phase transition in bulk.

- During phase transition, silver atoms showed liquid to $b c c$ to $f c c$ (with $h c p$ ) both in bulk and in confined region. However, bcc structure appears for a very short period, and as the simulation proceeds $b c c$ transformed into $f c c$ (or $h c p$ ).

- The interaction potential energy between graphene and silver influences the evolution of structural organization or phase transitions. At high interaction energy, silver depict good wettabilty in the confined region and phase transition occurs at a relatively higher temperature.

- Dimension-less aspect ratio $x$ is invariant at the early stage of tensile 


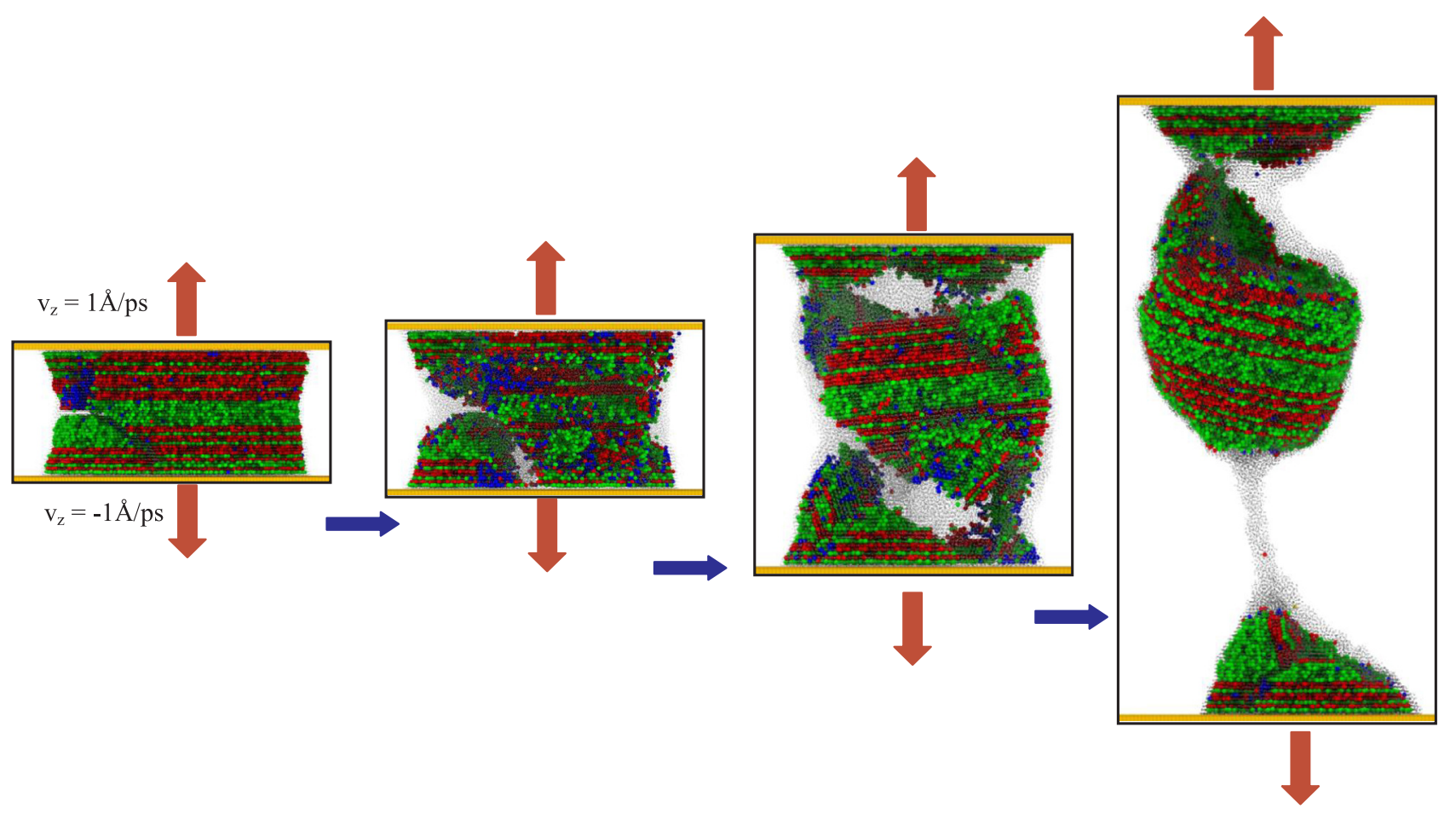

(a)

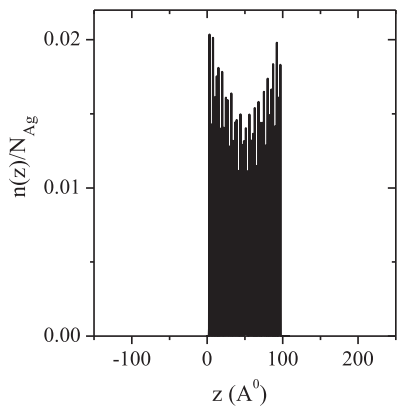

0 ps

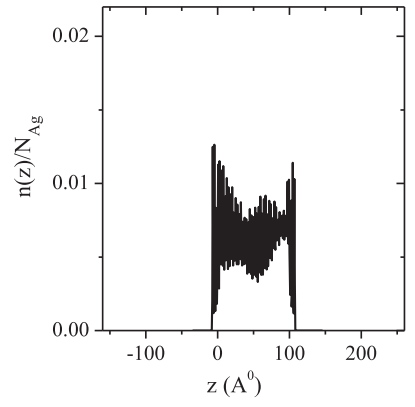

$10 \mathrm{ps}$

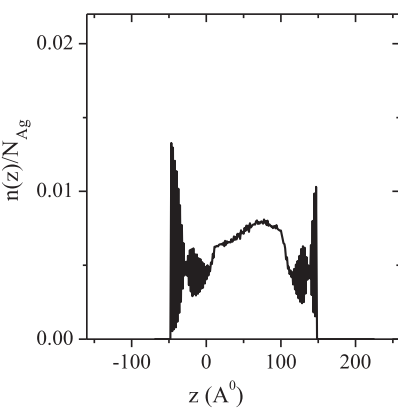

$50 \mathrm{ps}$

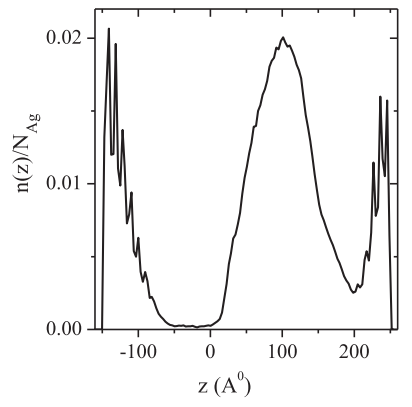

$150 \mathrm{ps}$

(b)

Fig. 10. The detachment of silver in confined region between two graphene sheets during tensile pulling of graphene sheet by vz $=1 \AA$ A ps and - $1 \AA ̊$ ps at $1200 \mathrm{~K}$ : (a) snapshots and (b) average number distribution, $\frac{n(z)}{N_{A g}}$, of silver in a confined region at various instant.

deformation, but it shows a sharp peak at the onset of detachment of silver from both graphene sheets.

The solidification and organization of silver nano-particle both in bulk and in the confined region will control overall properties of metal matrix nano-composite.

\section{Data availability}

The raw data required to reproduce these findings are available by request by author email: sunil@nmlindia.org. 


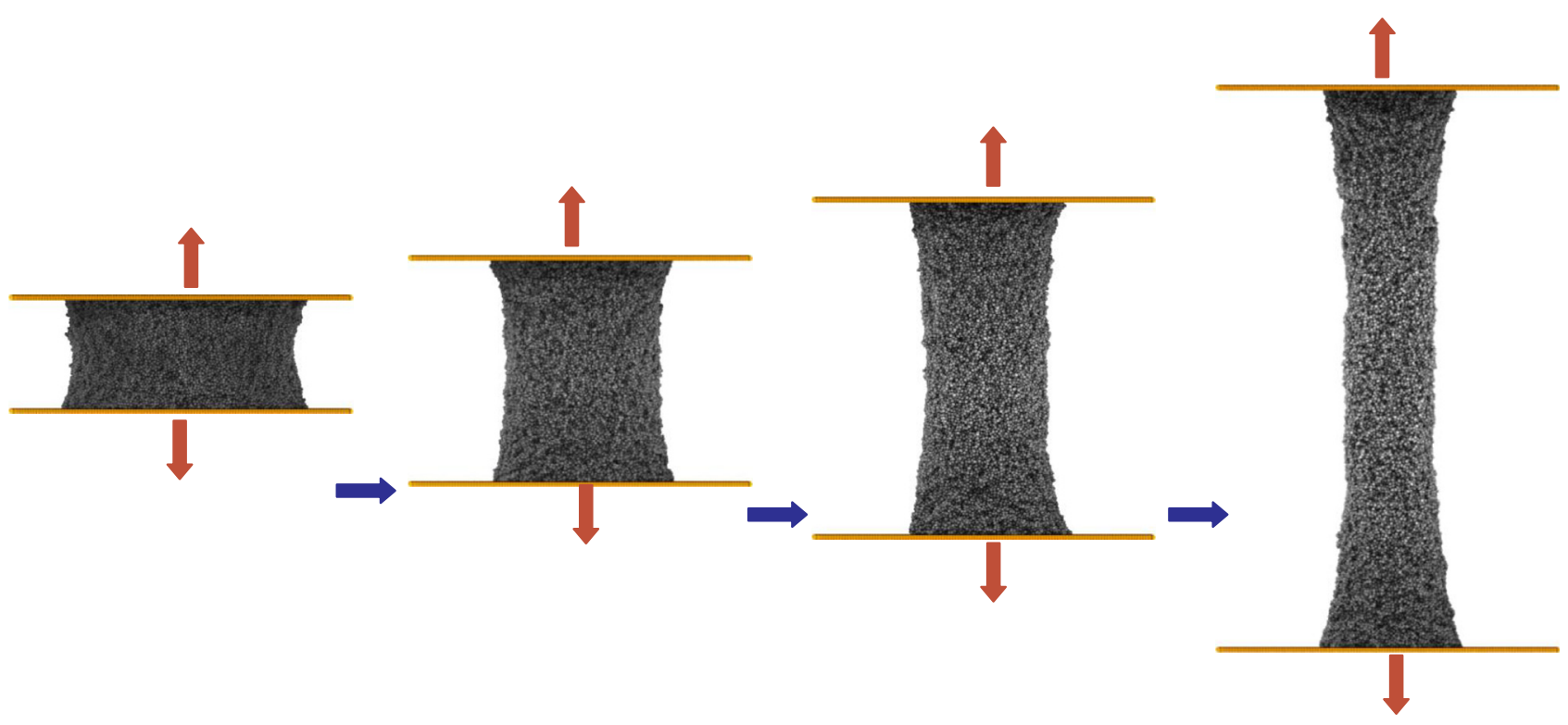

(a)

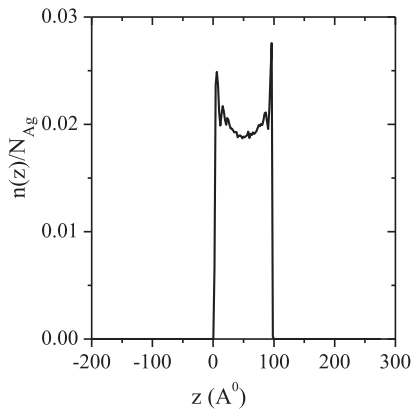

$0 \mathrm{ps}$

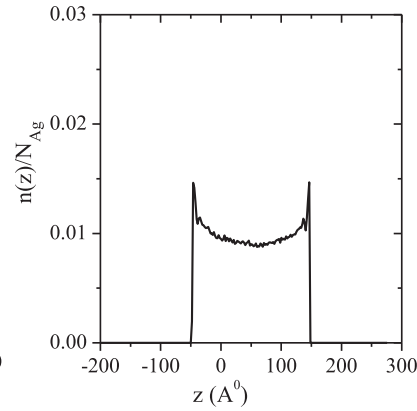

$50 \mathrm{ps}$

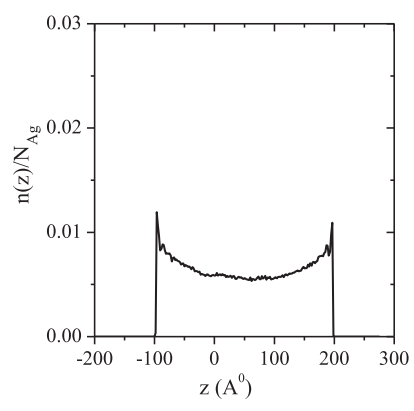

$100 \mathrm{ps}$

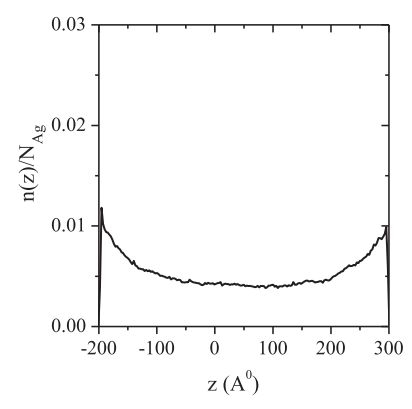

200ps

(b)

Fig. 11. Elongation of silver in confined region between two graphene sheets during tensile pulling of graphene sheet by vz $=1 \AA / \mathrm{ps}$ and $-1 \AA$ /ps at $1500 \mathrm{~K}$ : (a) snapshot and (b) average number distribution, of silver in a confined region. 


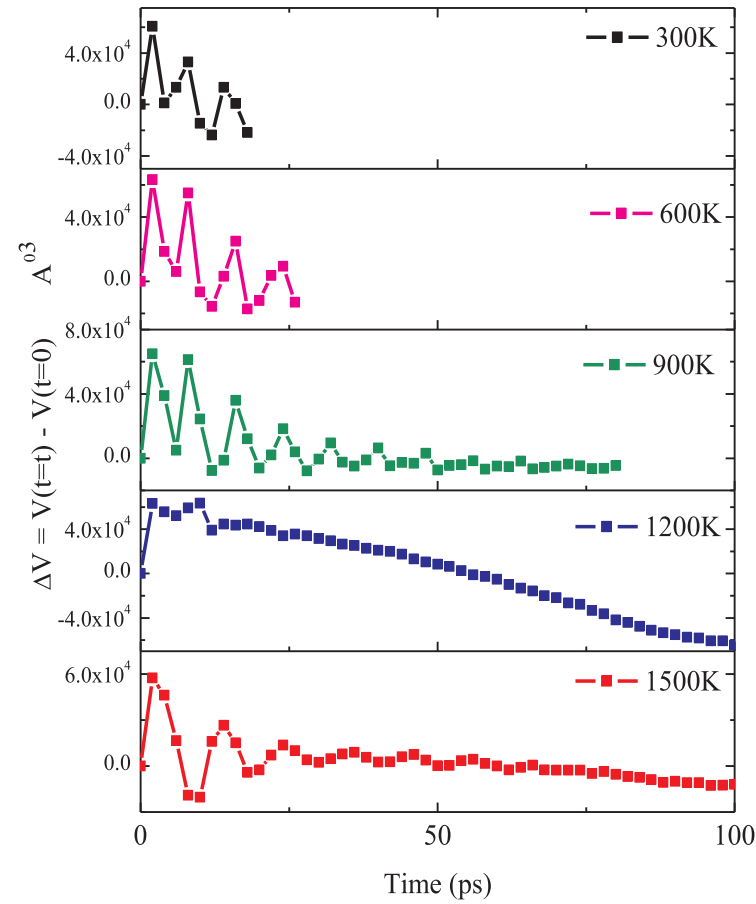

(a)

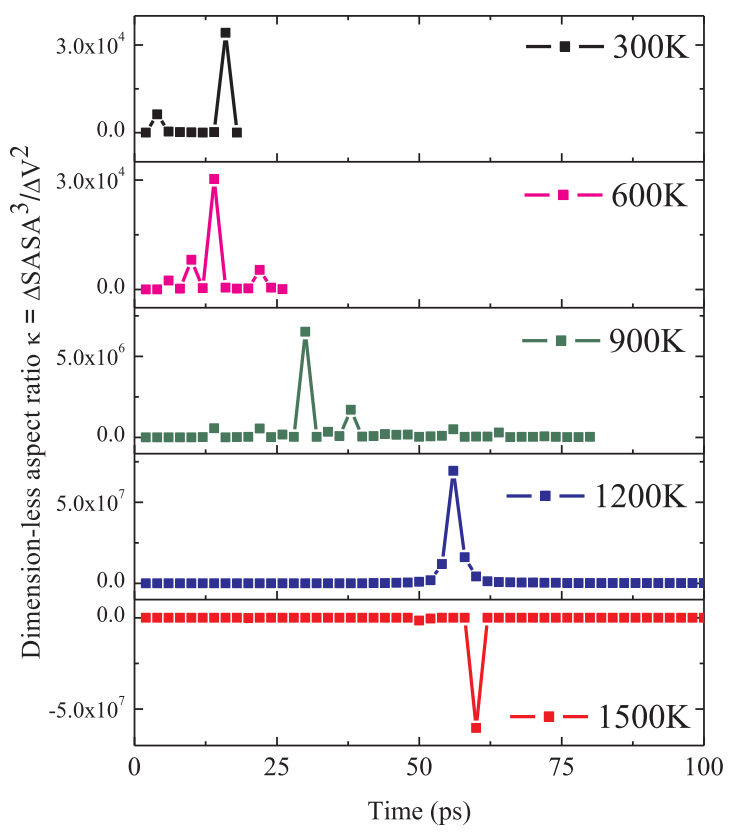

(c)

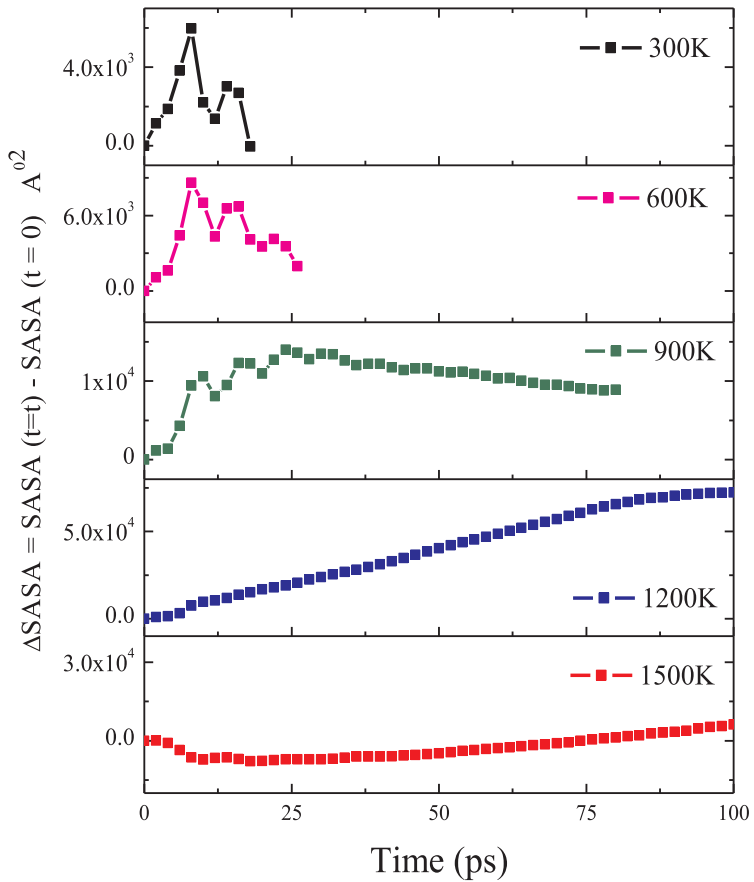

(b)

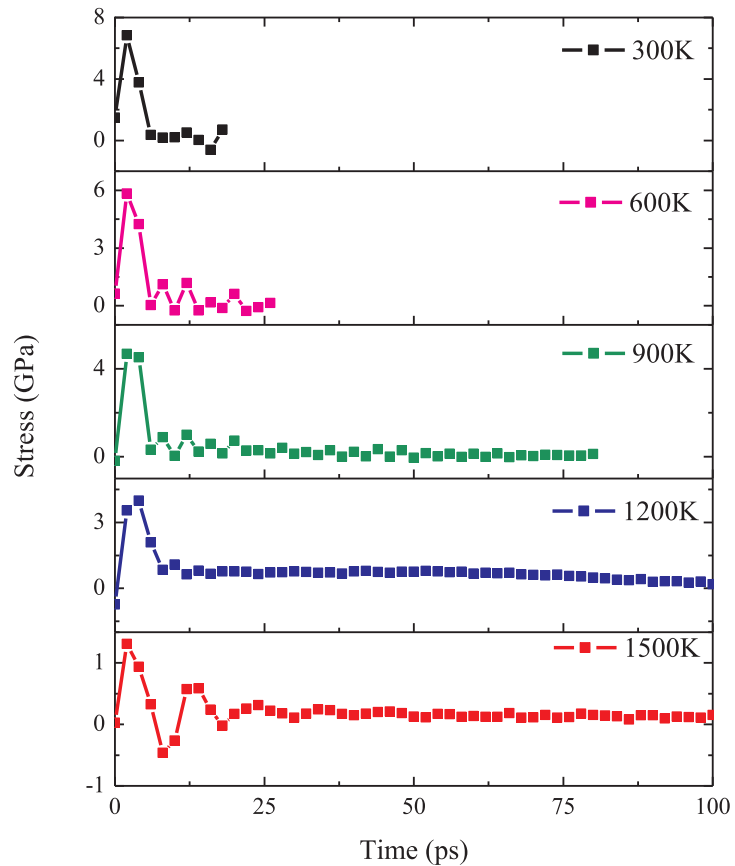

(d)

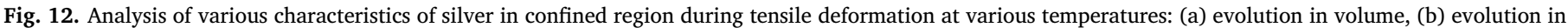
solvent accessible surface area, (c) dimension-less aspect ratio and (d) Stress. 


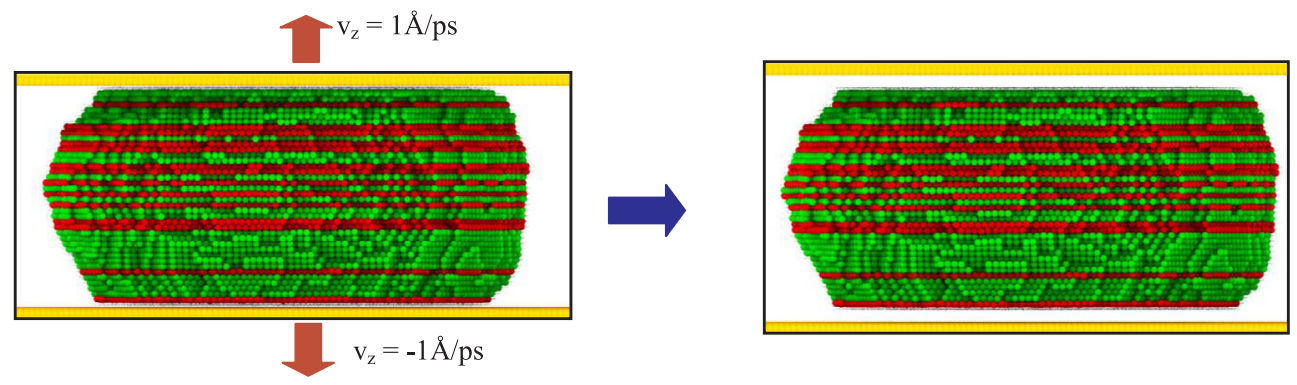

(a) $\varepsilon_{\mathrm{C}-\mathrm{Ag}}=0.01 \mathrm{eV}$
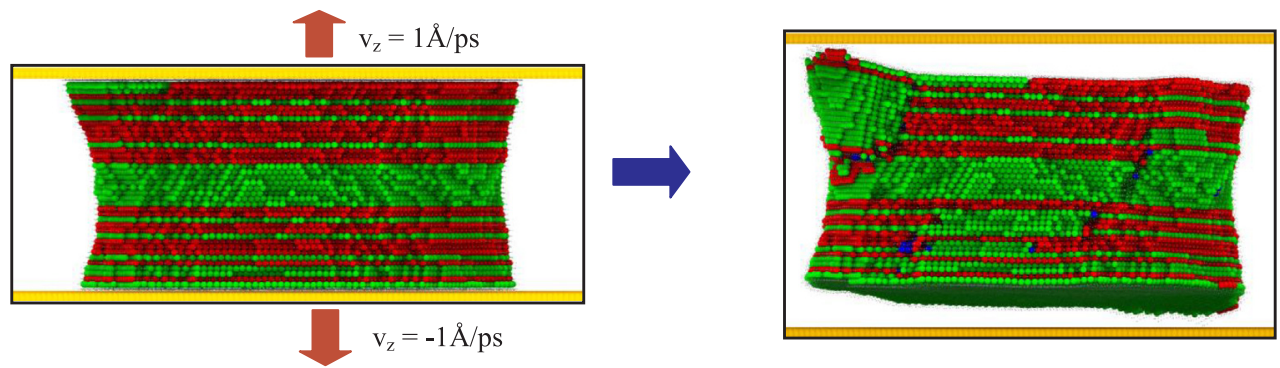

(b) $\varepsilon_{\mathrm{C}-\mathrm{Ag}}=0.03 \mathrm{eV}$
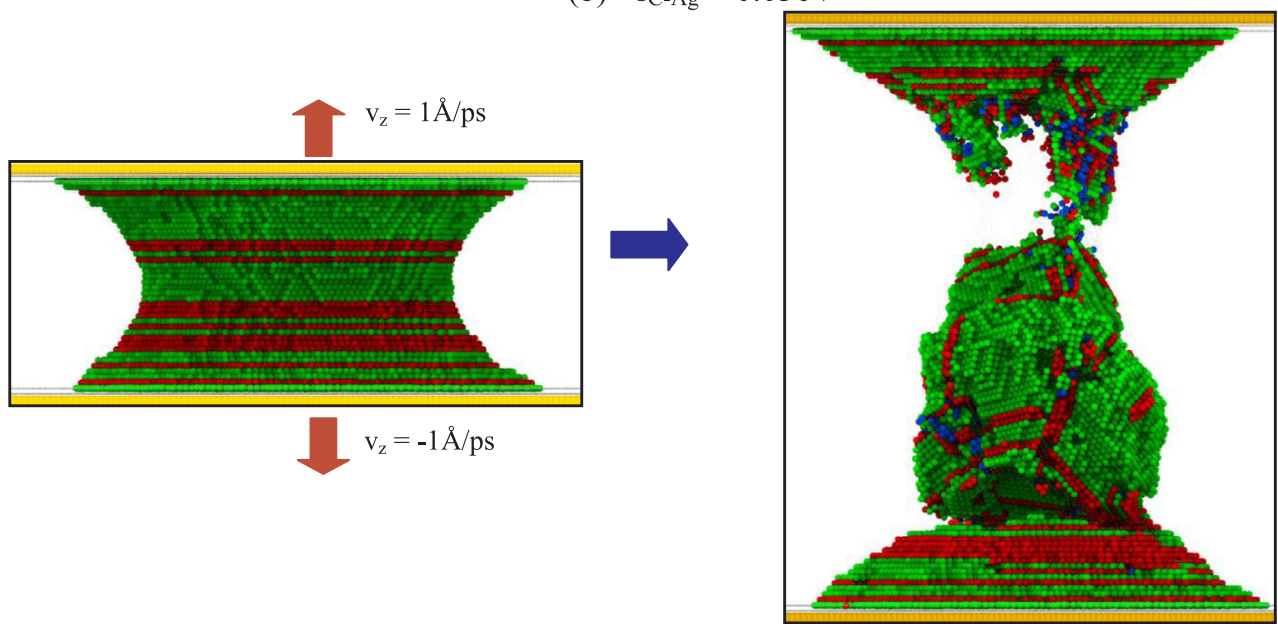

(c) $\varepsilon_{\mathrm{C}-\mathrm{Ag}}=0.05 \mathrm{eV}$

Fig. 13. Visuallization of detachment process for silver in confined region between two graphene sheets during tensile pulling of graphene sheet by vz $=1 \AA / p s$ and $-1 \AA / \mathrm{ps}$ at various values of $\varepsilon_{\mathrm{C}-\mathrm{Ag}}:$ (a) $\varepsilon_{\mathrm{C}-\mathrm{Ag}}=0.01 \mathrm{eV}$, (b) $\varepsilon_{\mathrm{C}-\mathrm{Ag}}=0.03 \mathrm{eV}$, and $\varepsilon_{\mathrm{C}-\mathrm{Ag}}=0.05 \mathrm{eV}$.

\section{References}

[1] C. Lee, X. Wei, J.W. Kysar, J. Hone, Measurement of the elastic properties and intrinsic strength of monolayer graphene, Science 321 (2008) 385-388.

[2] K.S. Novoselov, V.I. Fal, L. Colombo, P.R. Gellert, M.G. Schwab, K. Kim, A roadmap for graphene, Nature 490 (2012) 192-200.

[3] T. Wejrzanowski, M. Grybczuk, M. Chmielewski, K. Pietrzak, K.J. Kurzydlowski, A. Strojny-Nedza, Thermal conductivity of metal-graphene composites, Mat. Des. 99 (2016) 163-173.

[4] S. Sharma, P. Kumar, R. Chandra, Mechanical and thermal properties of graphene-carbon Nanotubes-reinforced metal matrix composites: a molecular dynamics study, J. Comp. Mat. 51 (2017) 3299-3313.

[5] J. Sun, J. Zhao, M. Chen, Y. Zhou, X. Ni, Z. Li, F. Gong, Multilayer graphene reinforced functionally graded tungsten carbide nano-composites, Mat. Des. 134 (2017) 171-180.

[6] K.F. Mak, C. Lee, J. Hone, J. Shan, T.F. Heinz, Atomically thin MoS 2: a new directgap semiconductor, Phys. Rev. Lett. 105 (2010) 136805.

[7] Y. Zhu, S. Murali, W. Cai, X. Li, J.W. Suk, J.R. Potts, R.S. Ruoff, Graphene and graphene oxide: synthesis, properties, and applications, Adv. Mat. 22 (2010) 3906-3924.

[8] G. Eda, M. Chhowalla, Chemically derived graphene oxide: towards large-area thinfilm electronics and optoelectronics, Adv. Mat. 22 (2010) 2392-2415.

[9] J. Li, C.Y. Liu, Ag/graphene heterostructures: synthesis, characterization and optical properties, Eur. J. Inorg. Chem. 1244-1248 (2010).

[10] H.W. Tien, Y.L. Huang, S.Y. Yang, J.Y. Wang, C.C.M. Ma, The production of graphene nano-sheets decorated with silver nano-particles for use in transparent, conductive films, Carbon 49 (2011) 1550-1560.

[11] M.R. Das, R.K. Sarma, R. Saikia, V.S. Kale, M.V. Shelke, P. Sengupta, Synthesis of silver nanoparticles in an aqueous suspension of graphene oxide sheets and its antimicrobial activity, Coll. Surf. B: Bio. 83 (2011) 16-22.

[12] L. Liu, J. Liu, Y. Wang, X. Yan, D.D. Sun, Facile synthesis of monodispersed silver nanoparticles on graphene oxide sheets with enhanced antibacterial activity, New J. Chem. 35 (2011) 1418-1423.

[13] A.F. de Faria, D.S.T. Martinez, S.M.M. Meira, A.C.M. de Moraes, A. Brandelli, A.G. Souza Filho, O.L. Alves, Anti-adhesion and antibacterial activity of silver nanoparticles supported on graphene oxide sheets, Coll. Surf. B: Bio. 113 (2014) $115-124$

[14] J. Hwang, T. Yoon, S.H. Jin, J. Lee, T.S. Kim, S.H. Hong, S. Jeon, Enhanced mechanical properties of graphene/copper nanocomposites using a molecular-level mixing process, Adv. Mat. 25 (2013) 6724-6729.

[15] M. Li, H. Che, X. Liu, S. Liang, H. Xie, Highly enhanced mechanical properties in Cu matrix composites reinforced with graphene decorated metallic nanoparticles, J. Mat. Sci. 49 (2014) 3725-3731.

[16] D.J. Guo, Z.Y. Wei, B. Shi, S.W. Wang, L.Z. Wang, W. Tan, S.M. Fang, Copper nanoparticles spaced 3D graphene films for binder-free lithium-storing electrodes, J. Mat. Chem. A 4 (2016) 8466-8477.

[17] N. Salam, A. Sinha, A.S. Roy, P. Mondal, N.R. Jana, S.M. Islam, Synthesis of silver-graphene nanocomposite and its catalytic application for the one-pot threecomponent coupling reaction and one-pot synthesis of 1, 4-disubstituted 1, 2, 3 triazoles in water, RSC Adv. 4 (2014) 10001-10012.

[18] S.K. Bhunia, N.R. Jana, Reduced graphene oxide-silver nanoparticle composite as 
visible light photocatalyst for degradation of colorless endocrine disruptors, ACS Appl. Mat. Int. 6 (2014) 20085-20092.

[19] J. Wang, Z. Li, G. Fan, H. Pan, Z. Chen, D. Zhang, Reinforcement with graphene nanosheets in aluminum matrix composites, Scr. Mat. 66 (2012) 594-597.

[20] S.F. Bartolucci, J. Paras, M.A. Rafiee, J. Rafiee, S. Lee, D. Kapoor, N. Koratkar, Graphene-aluminum nanocomposites, Mat. Sci. Eng.: A 528 (2011) 7933-7937.

[21] T.S. Koltsova, L.I. Nasibulina, I.V. Anoshkin, V.V. Mishin, E.I. Kauppinen, O.V. Tolochko, A.G. Nasibulin, New hybrid copper composite materials based on carbon nanostructures, J. Mat. Sci. Eng. B 2 (2012) 240-246.

[22] L.Y. Chen, H. Konishi, A. Fehrenbacher, C. Ma, J.Q. Xu, H. Choi, H.F. Xu, F.E. Pfefferkorn, X.C. Li, Novel nanoprocessing route for bulk graphene nanoplatelets reinforced metal matrix nanocomposites, Scr. Mat. 67 (2012) 29-32.

[23] M.N. Amal, R. Asgari, M.R. Tabar, The formation of atomic nanoclusters on graphene sheets, Nanotechnology 20 (2009) 135602.

[24] K. Duan, L. Li, Y. Hu, X. Wang, Enhanced interfacial strength of carbon nanotube/ copper nanocomposites via Ni-coating: Molecular-dynamics insights, Phys. E: Lowdim. Sys. Nano. 88 (2017) 259-264.

[25] M.P. Allen, D.J. Tildesley, Computer Simulation of Liquids, Oxford University Press, 1989.

[26] D.C. Rapaport, R.L. Blumberg, S.R. McKay, W. Christian, The art of molecular dynamics simulation, Comp. Phys. 10 (1996) 456456.

[27] G. Ciccotti, M. Ferrario, C. Schuette, Molecular dynamics simulation, Entropy 16 (2014) 233.

[28] G.J. Ackland, G. Tichy, V. Vitek, M.W. Finnis, Simple Nbody potentials for the noble metals and nickel, Philos. Mag. A 56 (1987) 735-756.

[29] H. Akbarzadeh, H. Yaghoubi, Molecular dynamics simulationsof silver nanocluster supported on carbon nanotubes, J. ColloidInterface Sci. 418 (2014) 178-184.

[30] L. Verlet, Computer, "experiments" on classical fluids. I.thermodynamical properties of Lennard-Jones molecules, Phys. Rev. 159 (1967) 98-102.

[31] G.J. Martyna, D.J. Tobias, M.L. Klein, Constant pressure molecular dynamics algorithms, J. Chem. Phys. 101 (1994) 4177-4189.

[32] M. Parrinello, A. Rahman, Polymorphic transitions in single crystals: a new molecular dynamics method, J. Appl. Phys. 52 (1981) 7182-7190.

[33] S. Plimpton, Fast parallel algorithms for short-range molecular dynamics, J. Comput. Phys. 117 (1995) 1-19.

[34] A. Stukowski, Visualization and analysis of atomistic simulation data with OVITOthe open visualization Tool, Mod. Sim. Mat. Sci. Eng. 18 (2010) 015012.

[35] A. Stukowski, Structure identification methods for atomisticsimulations of crystalline materials, Mod. Sim. Mat. Sci. Eng. 20 (2012) 045021.

[36] W. Humphrey, A. Dalke, K. Schulten, VMD: visual molecular dynamics, J. Mol. Grap. 14 (1996) 33.
[37] H.-L. Chen, S.-P. Ju, S. Wang, C.-T. Pan, C.-W. Huang, Size-dependent thermal behaviors of five-fold twinned silver nanowires: a computational study, J. Phys. Chem. C 120 (2016) 12840-12849.

[38] A.E. Galashev, Computer stability test for aluminum films heated on a graphene sheet, Tech. Phys. 59 (2014) 467-473.

[39] S. Sarkar, P.K. Das, Thermal and structural stability of singleand multi-walled carbon nanotubes up to $1800^{\circ} \mathrm{C}$ in argon studied by raman spectroscopy and transmission electron microscopy, Mat. Res. Bull. 48 (2013) 41-47.

[40] B.G. Levine, J.E. Stone, A. Kohlmeyer, Fast analysis of molecular dynamics trajectories with graphics processing unit's radial distribution function histogramming, J. Comp. Phys. 230 (2011) 3556-3569.

[41] B. Shen, C.Y. Liu, Y. Jia, G.Q. Yue, F.S. Ke, H.B. Zhao, L.Y. Chen, S.Y. Wang, C.Z. Wang, K.-M. Ho, Molecular dynamics simulation studies of structural and dynamical properties of rapidly quenched Al, J. Non-Cryst. Solids 383 (2014) 13-20.

[42] H. Tsuzuki, P.S. Branicio, J.P. Rino, Structural characterization of deformed crystals by analysis of common atomic neighborhood, Comp. Phys. Comm. 177 (2007) 518-523.

[43] D. Faken, H. Jonsson, Systematic analysis of local atomic structure combined with 3D computer graphics, Comp. Mat. Sci. 2 (1994) 279-286.

[44] J.D. Honeycutt, H.C. Andersen, Molecular dynamics study of melting and freezing of small Lennard-Jones clusters, J. Phys. Chem. 91 (1987) 4950-4963.

[45] S. Kumar, S.K. Pattanayek, G.G. Pereira, Polymers encapsulated in short single wall carbon nanotubes: Pseudo-1D morphologies and induced chirality, J. Chem. Phys. 142 (2015) 114901.

[46] S. Kumar, Effect of applied force and atomic organization of copper on its adhesion to a graphene substrate, RSC Adv. 7 (2017) 25118-25131.

[47] M.S. Daw, S.M. Foiles, M.I. Baskes, The embedded-atom method: a review of theory and applications, Mat. Sci. Rep. 9 (1993) 251-310.

[48] Z.-A. Tian, L. Rang-Su, P. Ping, H. Zhao-Yang, L. Hai-Rong, Z. Cai-Xing, D. Ke-Jun, Y. Ai-Bing, Freezing structures of free silver nanodroplets: a molecular dynamics simulation study, Phys. Lett. A 373 (2009) 1667-1671.

[49] S. Kumar, V.C. Srivastava, G.K. Mandal, S.K. Pattanayek, K.L. Sahoo, Single-walled carbon nanotube engendered pseudo-1D morphologies of silver nanowire, J. Phys. Chem. C 121 (2017) 20468-20480.

[50] J. Sun, L. He, Y.C. Lo, T. Xu, H. Bi, L. Sun, Z. Zhang, S.X. Mao, J. Li, Liquid-like pseudoelasticity of sub-10-nm crystalline silver particles, Nat. Mat. 2014 (13) (2014) 1007.

[51] Z.A. Tian, R.S. Liu, C.X. Zheng, H.R. Liu, Z.Y. Hou, P. Peng, Formation and evolution of metastable bcc phase during solidification of liquid Ag: a molecular dynamics simulation study, J. Phys. Chem. A 112 (2008) 12326-12336. 